\title{
Review article: Performance measures of parallel kinematics manipulators
}

\author{
Abdur Rosyid, Bashar El-Khasawneh, and Anas Alazzam \\ Mechanical Engineering Department, Khalifa University of Science and Technology, \\ P.O. Box 127788, Abu Dhabi, United Arab Emirates \\ Correspondence: Bashar El-Khasawneh (bashar.khasawneh@ku.ac.ae)
}

Received: 3 February 2019 - Revised: 14 December 2019 - Accepted: 19 January 2020 - Published: 16 March 2020

\begin{abstract}
This extensive review paper, which involves 204 papers, discusses comprehensively a number of performance indices that are instrumental in the design of parallel kinematics manipulators. These indices measure the workspace as well as its quality including the distance to singularity, dexterity, manipulability, force transmission, accuracy, stiffness, and dynamic performance. After being classified, the indices are discussed in terms of some important aspects including definition, physical meaning soundness, dependency, consistency, scope of applicability, and computation cost. For the sake of completeness, some key mathematical expressions of the indices are provided.
\end{abstract}

\section{Introduction}

Parallel kinematics manipulators (PKMs) have been extensively studied in the last two decades with a few of them deployed into commercial market particularly for demanding tasks such as high-speed pick-and-place and aircraft component machining as they offer high dynamics, high agility, high payload-to-weight ratio, distributed joint errors, and simple inverse kinematics. Nevertheless, they are also attributed with some drawbacks such as smaller workspace, singularity issue, and complicated forward kinematics. To optimize the performance of a PKM, some performance measures or performance indices are usually evaluated and optimized during the design phase.

Patel and Sobh (2015) categorized the performance measures into either local or global, kinematic or dynamic, and intrinsic or extrinsic. The local measures depend on the manipulator posture whereas the global measures evaluate the manipulator performance across the whole workspace. The global performance index GPI can be obtained by analytically averaging the local performance index $P$ over the workspace $W$ as given by Eq. (1). If the local performance index cannot be expressed analytically in terms of the workspace, Eq. (2) can be used to perform discrete averaging. In the latter case, the number of points $N$ being evaluated may affect the accuracy of the averaging. The evaluation of a large number of points can be computationally expensive. In such a case, less representative sampling points can be used.

$$
\begin{aligned}
& \mathrm{GPI}=\frac{\int_{W} P \cdot \mathrm{d} W}{\int_{W} \mathrm{~d} W} \\
& \mathrm{GPI}=\frac{1}{N} \sum_{i=1}^{N} P_{i}
\end{aligned}
$$

To evaluate the performance index $P$ in a single cross-section of the manipulator workspace, section performance index SPI (Wang et al., 2014) has been proposed and is given by:

$\mathrm{SPI}=\frac{\int_{S} P \cdot \mathrm{d} S}{\int_{S} \mathrm{~d} S}$

where $S$ denotes the cross-section area of the workspace.

Furthermore, Zhang et al. (2014) proposed the use of a global performance index which evaluates the distribution characteristics of a performance index, including central tendency, dispersion, and shape. This global index integrates four performance indices: global average index (GAI), global volatility index (GVI), global skewness index (GSI), and global kurtosis index (GKI).

The kinematic measures evaluate the kinematic performance of the manipulator whereas the dynamic measures 
indicate the dynamic properties of the manipulator. The intrinsic measures indicate the inherent characteristics of the manipulator regardless of its task whereas the extrinsic measures are related to the manipulator task. From the viewpoint of workspace, the performance measures can be classified into two categories (Lou et al., 2014): (i) measures on workspace geometric properties, i.e. area/volume and shape, and (ii) measures on workspace quality such as distance to singularity, kinematic dexterity, manipulability, transmissibility, accuracy, repeatability, stiffness, dynamic dexterity, etc.

A discussion on well-known classical performance indices can be found in Merlet (2006c). To the authors' knowledge, the most recent and comprehensive review on the manipulator performance measures is Patel and Sobh (2015). Although the paper discusses a good number of indices, it does not include some important indices for parallel kinematics manipulators such as stiffness indices. In this paper, a comprehensive review of a number of performance indices for PKMs is presented. More focus is given to indices commonly considered in the design of PKMs. In fact, some of the indices are borrowed or extended from those for serial kinematics manipulators (SKMs) while some others have been developed from the beginning for PKMs. The development of the indices to the current state of the art along with the description of their advantages as well as limitations and disadvantages is presented. Some key mathematical expressions are included to give a self-contained, clearer description on the indices. However, a reader interested in more details should refer to corresponding references.

This paper is a major extension of the authors' shorter review previously published (Rosyid et al., 2017) with the following differences. First, while the previous paper only discusses the kinematic performance measures, this paper also includes the dynamic performance measures. Furthermore, more kinematic performance measures are introduced in this paper for more completeness. In particular, force transmission performance measures are very little discussed in the previous review but they are extensively discussed in this paper. Second, this paper includes key mathematical expressions of most of the reviewed performance indices along with their description while the previous paper only provides a concise description of the indices and does not include the mathematical expressions of the indices. Third, a new classification of the performance measures is used in this paper to improve the taxonomy of the performance measures. Fourth, more discussions and insights on the performance measures are provided in this paper. Furthermore, some more recommendations are added in this paper for future research direction. Finally, as a result of the more completeness of this paper, the number of relevant references are significantly increased.

\section{Workspace}

The workspace is the set of points (locations) the end effector can reach. It is determined by the manipulator's mobility which is represented by the number and types of its degrees of freedom (DOF), and constrained by the link lengths, the joint limits, and the interference between the components. The workspace is usually evaluated in terms of its size (area/volume) and shape. The latter is commonly quantified by the aspect ratio of the regular workspace. In general, it is preferred to have larger and better-shaped workspace. The workspace can also be characterized by using workspace volume index (Merlet, 2006a), space utilization index (Lee et al., 2010), or footprint ratio (Shijun, 2015) which are all defined as the ratio between the workspace volume and the volume of the machine. Recently, a normalized index bounded between 0 and 1 called global workspace conditioning index (GWCI) (Enferadi and Nikrooz, 2017) has been introduced and applied in a spherical PKM. This index is given by the workspace volume divided by the product of motion ranges in each degree of freedom.

Prior to optimizing the geometrical parameters in order to achieve better workspace, selecting an appropriate topology is crucial. Many manipulators, such as Hexaglide, Linapod, Pentaglide, sliding H4, and Triaglide, have sliders (gliders) to provide a larger workspace.

The manipulator workspace is commonly classified into several types (Merlet, 2006a) which include constantorientation workspace (translation workspace), orientation workspace, reachable workspace (maximal workspace), inclusive workspace, dexterous workspace, and total orientation workspace. Useful workspace, sometimes also called used workspace, which indicates part of the workspace to be used for a specific application is usually a regular workspace such as a rectangle, circle, cuboid, sphere, or cylinder. A designer is often much interested in the useful workspace as it indicates part of the workspace which can be really utilized for the application. Baek et al. (2001) presented a method to find maximally inscribed rectangle in the workspace of SKMs and PKMs. Furthermore, Bonev and Ryu (2001) introduced a two-dimensional subspace of orientation workspace called projected orientation workspace and defined it as the set of possible directions of the approach vector of the moving platform.

The workspace can be represented completely in sixdimensional space. However, a graphical representation is only possible up to three-dimensional space. Therefore, the position workspace is usually represented separately from the orientation workspace. A two-dimensional plot can be used to show the workspace of a planar manipulator, whereas a three-dimensional plot can be utilized to represent the workspace of a spherical or spatial manipulator. The two-dimensional workspace plot can be presented in either Cartesian or polar coordinate system whereas the threedimensional workspace plot can be presented in Cartesian, 
cylindrical, or spherical coordinate system. In general, the workspace of a manipulator with more than 3 DOF can be graphically illustrated by fixing $(n-3)$ DOF. Depending on which DOF to be fixed, the workspace will be different (Merlet, 2006a). Furthermore, while plotting the workspace of SKMs is straight forward, that is typically not the case for PKMs.

Generally, three main approaches namely geometrical approach, discretization numerical approach, and nondiscretization numerical approach can be used to determine and plot the workspace. The geometrical approach has been extensively used in the early works on the workspace determination of PKMs. Using the geometrical approach, Bajpai and Roth (1986) studied the workspace of PKMs and the effects of legs' length to the workspace. Three years later, Gosselin (1989) presented his work on the determination of constant-orientation workspace of PKMs. Subsequently, Merlet (1995) presented the determination of orientation workspace of PKMs. Afterwards, Kim et al. (1997) proposed an algorithm to determine the reachable and dexterous workspace of PKMs. As planar PKMs need different treatments, Gosselin and Jean (1996) followed by Merlet et al. (1998) investigated the workspace determination of planar PKMs.

In the geometrical approach, the true boundaries of a PKM workspace can be quickly and accurately obtained from the intersection of the boundaries of every open-loop chains composing the PKM. The use of computer-aided design (CAD) software can make this process easier and faster such as the work proposed by Arrouk et al. (2010). Nevertheless, this approach lacks general applicability as it usually should be tailored to the considered manipulator. In addition, it is difficult to include all the constraints by using this approach.

In the discretization numerical approach, a discretized bounding space covering all possible points in the workspace is created. Subsequently, the inverse kinematics along with the constraints is used to check whether or not a possible point belongs to the workspace. This approach is sometimes called binary representation since a reachable point is usually indicated by " 1 " and plotted whereas an unreachable point is indicated by " 0 " and not plotted. This approach is intuitive, applicable to all types of PKMs, and able to include all the constraints. However, the size of the discretization steps affects the accuracy of this approach. In addition, small voids inside the workspace cannot be detected unless the discretization steps are small enough to capture them. Monte Carlo method (Alciatore and Ng, 1994; Rastegar and Perel, 1990) is similar to this approach as a large number of random, discrete active joint points within the joint range are input to the forward kinematics and accordingly the end effector position points are plotted. However, forward kinematics for PKMs with the exception of the very simple ones is typically complicated. Therefore, Monte Carlo approach is also conducted in the other direction where a large number of ran- dom, discrete possible workspace points in a bounding space are transformed to the joint positions by using the inverse kinematics. If a joint position falls within the joint range and meets the constraints, the corresponding workspace point is reachable.

Some recent works using the discretization numerical approach include a new approach proposed by Bonev and Ryu (2001) to determine the three-dimensional orientation workspace of 6-DOF PKMs. An algorithm based on the discretization approach was proposed by Castelli et al. (2008) to determine the workspace, the workspace boundaries, the workspace volume, and the workspace shape index of SKMs and PKMs. A discretization method to determine the reachable workspace and detect available voids of PKM was presented by Dash et al. (2005).

Beside the aforementioned two approaches, several nondiscretization numerical methods have been proposed to determine the workspace of PKMs. Some of the methods are as follows (Merlet, 2006a; Wang et al., 2001):

- Jacobian rank deficiency method (Jo and Haug, 1989b) which is only practical to determine constant orientation workspace.

- Numerical continuation method (Haug et al., 1995; Jo and Haug, 1989a) which can avoid singularity points but is only practical to determine constant orientation workspace.

- Constrained optimization method (Snyman et al., 2000) which is modified from the numerical continuation method.

- Boundary search method (Wang et al., 2001) which is based on constrained non-linear programming.

- The principle that the velocity vector of the moving platform cannot have a component along the normal of the boundary (Kumar, 1992), but this method cannot be applied to manipulators with prismatic joints and cannot easily include the mechanical limits and interference between links.

- Interval analysis method (Kaloorazi et al., 2014) which can handle almost any constraints and any number of DOF.

Recently, Bohigas et al. (2012) proposed branch-andprune technique to determine all the workspace boundary points of general lower-mobility (3-DOF or lower) SKMs and PKMs. This technique overcomes the limitation of numerical continuation method. Gao and Zhang (2011) presented Simplified Boundary Searching (SBS) method which integrates geometrical approach, discretization method, and inverse kinematics model of a PKM. Saputra et al. (2014) proposed swarm optimization approach to determine the workspace of PKM. Finally, Zhou et al. (2017) proposed 
a novel numerical approach to determine simultaneously both position workspace and orientation workspace for both lower-mobility and six-DOF PKMs.

\section{Jacobian matrix and dependency issues}

The Jacobian matrix relates the velocities of the moving platform to the velocities of the active joints. Furthermore, it also relates the active joint wrench to the task wrench. It is discussed here because it serves as the base of many performance measures.

The velocity kinematics of the manipulator can be expressed by:

$$
\mathbf{A} \dot{\boldsymbol{q}}+\mathbf{B} \dot{\boldsymbol{x}}=0
$$

where $\dot{\boldsymbol{x}}$ is the end effector velocity and $\dot{\boldsymbol{q}}$ is the actuator velocity. The matrices $\mathbf{A}$ and $\mathbf{B}$ are called the forward and inverse Jacobian matrices, respectively. The total Jacobian matrix $\mathbf{J}$ can be defined as:

$\mathbf{J}=-\mathbf{B}^{-1} \mathbf{A}$

The properties of the Jacobian matrix, unfortunately, is dependent on the scale and frame. Dependency on the scale means that the value is significantly affected by the choice of unit. Changing the unit can dramatically change the values of its properties (Yu et al., 2008). Furthermore, the Jacobian matrix is not invariant with respect to the reference frame used (Doty et al., 1995).

Furthermore, when a manipulator mixes translational and rotational DOF, the Jacobian matrix becomes unitinconsistent (non-homogeneous) and accordingly lacks sound physical meanings (Lipkin and Duffy, 1988). To deal with this issue, several techniques have been proposed to normalize inconsistent (non-homogeneous) Jacobian matrix including the following:

- Using characteristic length or natural length (Angeles, 2002; Ma and Angeles, 1991b; Ranjbaran et al., 1995; Fattah and Hasan Ghasemi, 2002; Angeles, 2006; Tandirci et al., 1992; Chablat et al., 2002): All Jacobian matrix components having units of length are divided by a characteristic length. The characteristic length giving the best performance is called the natural length by Ma and Angeles (1991b). The characteristic length can be derived based on the evaluation of manipulator isotropy (Chablat et al., 2002; Chablat and Angeles, 2002). In case it is not derivable, a common practice is dividing the components having units of length by the average platform radius or the platform radius of the corresponding limb. Despite the wide use of the characteristic length, its geometric interpretation is not straightforward. To deal with this issue, the use of homogeneous space (Khan and Angeles, 2006) reveals a more direct geometric interpretation of the characteristic length.
- Using scaling matrix: Stocco et al. $(1998,1999)$ proposed the concept of scaling matrix which includes taskspace scaling matrix, which can be considered a generalized extension of the characteristic length, and jointspace scaling matrix. Another concept of scaling matrix has been proposed in Lou et al. (2004) where the distances between the center of the moving platform to the joints connecting the limbs to the moving platform are used in the scaling matrix.

- Using weighting factor (Hosseini et al., 2011; Hosseini and Daniali, 2011): The Jacobian matrix components having units of length are divided by a length (as in characteristic length method) and at the same time the corresponding velocity coordinates are multiplied by the same length. Moreover, different weights can be used for different coordinates. Both the length and the weights are constant across the workspace.

- Using velocities of three points on the moving platform (Pond and Carretero, 2006, 2007; Kim and Ryu, 2003; Kong et al., 2007): As a continuation of Gosselin's work (Gosselin, 1990a) which proposed the use of two points on the end effector to get homogeneous Jacobian matrix, the use of distinct and non-collinear three points on the moving platform leads to differential kinematic expressions giving dimensionless Jacobian matrix.

- Point-based method (Altuzarra et al., 2006; Gosselin, 1992; Altuzarra et al., 2008): This method is based on computational velocity formulation which can easily incorporate all kinematic variables in the manipulator and is suitable for general-purpose computational kinematics software. The Jacobian matrix obtained is dimensionless.

- General and systematic method (Liu et al., 2011): Giving $f \times f$ homogeneous Jacobian matrix, this method is implemented by firstly formulating the linear map between the joint rates and velocity twist using the generalized Jacobian and subsequently generating a linear map between the velocity twist and $f$ linear velocities at a set of selected points on the end-effector. However, this method works only for manipulators with one type of actuators.

- Homogeneous extended Jacobian matrix (Nurahmi and Caro, 2016): This method has been formulated for nonredundant manipulators by considering a set of linear independent axes at the points of a tetrahedron, which represents the permitted motions and the restricted motions of the moving platform.

\section{Singularity}

Singularity inside the workspace is a common drawback of PKMs, and therefore should be evaluated at the design stage. 
It has a very important role since most of the performance measures such as dexterity, manipulability, transmissibility, and stiffness are related to the singularity. Therefore, it has attracted much attention and accordingly various works have been conducted to characterize different types of singularities. A broad classification of singularities has been made by Ma and Angeles (1991a) who introduced three types of singularities, namely architectural singularity, configuration singularity, and formulation singularity. The architectural (or structural) singularity is a permanent singularity occurring inside the whole or a part of a manipulator workspace, caused by a particular architecture of a manipulator. This is the worst type of singularity. This type of singularity leads to so-called self-motion or sometimes called the Borel-Bricard motion. Karger and Husty (1998) and Karger (2008) studied all self-motions in a Stewart-Gough PKM. Wohlhart (2003, 2010) termed this type of singularity as architectural shakiness and pointed out that it means more than mere singularity; it means "architectural mobility". Moreover, he introduced degree of shakiness (Wohlhart, 2010) and pointed out that a PKM is architecturally mobile if it is shaky to the fourth degree, i.e. it is mobile if we start with locked active joints from any architecturally possible position and hence it is uncontrollable.

The configuration singularity is caused by a particular configuration (posture) of a manipulator. Most of the discussions on singularity are on this type of singularity. The formulation singularity is caused by the failure of a kinematic model at a particular posture of a manipulator, such as gimbal-lock when using a particular Euler angle representation. This type of singularity can be overcome by changing the kinematic model. It is worth mentioning that the architecture singularity overrides the configuration singularity whereas the configuration singularity overrides the formulation singularity.

In general, singular configurations can be determined either analytically or geometrically. A well-known analytical method was introduced by Gosselin and Angeles (1990) who evaluate singularity by observing the Jacobian matrices. They classified the singularity in PKMs into the following three types:

- Type 1 singularity (also called: direct kinematic singularity, forward kinematic singularity, serial singularity, or sub-mobility). Mathematically, this is indicated by the singularity of the forward Jacobian matrix $\mathbf{A}$. In this singularity, some velocities of the end-effector cannot be generated. In other words, very small changes in the joint space do not affect the end-effector pose. Hence, the manipulator loses one or more degrees of freedom.

- Type 2 singularity (also called: inverse kinematic singularity, parallel singularity, or over-mobility). Mathematically, this is indicated by the singularity of the inverse Jacobian matrix B. In this singularity, the end-effector can move although the joints are locked. Hence, the ma- nipulator gains one or more uncontrollable DOF. Accordingly, the stiffness of the PKM is locally lost.

- Type 3 singularity (also called: combined singularity). Mathematically, this is indicated by the singularity of both the forward Jacobian matrix $\mathbf{A}$ and the inverse Jacobian matrix $\mathbf{B}$. In this singularity, the end-effector can move when the joints are locked, and at the same time, the end-effector pose does not change due to very small changes in the joints. Ma and Angeles (1991a) pointed out that this type of singularity is a case of architecture singularity because of its design-dependence feature. However, the architecture singularity is not only this type of singularity.

A more general discussion on singularities was delivered by Zlatanov et al. (1995) who included the passive joints in the singularity evaluation. Based on differential kinematics equations which involve the passive joints, they defined a configuration as singular when the kinematics of the mechanism is indeterminate with respect to either the input or output velocities. In other words, a configuration is singular if either the forward or inverse kinematics problem does not have a general solution. Moreover, they classified singularities into six types based on the physical phenomena which occur in singular configurations. The six types are: (i) redundant input (RI) singularity which corresponds to serial singularity, (ii) redundant output $(\mathrm{RO})$ singularity which covers parallel singularity, (iii) impossible input (II) singularity, (iv) impossible output (IO) singularity, (v) increased instantaneous mobility (IIM) singularity, and (vi) redundant passive motion (RPM) singularity. In addition, there are 21 possible combinations of the six singularity types. Although the method is a general framework, it was developed only for non-redundant PKMs. Later, Zlatanov et al. (2002) introduced constraint singularity in lower mobility PKMs, which occurs when the screw system, formed by the constraint wrenches in all legs, loses rank. They pointed out that the constraint singularity is an IIM singularity, but not every IIM configuration is a constraint singularity. Merlet (2006a) pointed out that the architectural singularity can be seen as a special case of constraint singularities in which the motions of the end-effector are finite.

Using a geometric approach, Merlet (2016, 2006a) studied singularity of PKMs based on Grassmann line geometry and applied the method to various classes of PKMs. A generalized classification based on a geometric framework was made by Park and Kim (1999) who classified singularities into three types. The three types are: (i) configuration space singularity which occurs when the joint configuration space manifold is singular, (ii) actuator singularity which occurs at configurations where the mechanism loses one or more DOF as a result of the choice of actuated joints, and (iii) endeffector singularity which occurs when the moving platform loses DOF of an available motion. It is also possible that more than one of the above three types holds. This classi- 
fication is frame-independent and works also for a redundant case, including actuation redundancy. Following Park's classification, Liu et al. (2003) presented a thorough geometric treatment for each of the three singularity types. They also discussed second order singularities namely degenerate singularity and non-degenerate singularity which hold for each of the three singularity types. The degenerate singularity applies if it is continuous whereas the non-degenerate applies if it is isolated (Liu et al., 2003). A degenerate actuator singularity corresponds to a configuration where some of the links can move although all the actuators are locked, whereas a non-degenerate actuator singularity corresponds to configurations where certain actuator forces may cause the mechanism to break apart (Park and Kim, 1999).

Huang et al. (2013) classified the singularities of a Stewart-Gough PKM into seven types based on the physical kinematic status: (i) dead-point singularity, (ii) extremedisplacement singularity, (iii) constraint-dependency singularity, (iv) full-cycle geometry singularity, (v) instantaneous mobility increase, (vi) mobility transfer to a local, and (vii) variety-mobility-property singularity. Yuefa and Tsai (2016) classified singularities for lower mobility PKMs into three types: (i) limb singularity which occurs when the limb twist system degenerates and accordingly the moving platform loses one or more DOF, (ii) platform singularity which occurs when the overall wrench system of the moving platform degenerates and accordingly the moving platform gains one or more DOF, and (iii) actuation singularity which occurs when inappropriate joints are chosen as the active joints and hence the moving platform still possesses certain DOF although all the actuators are locked. Recently, Chen et al. (2015b) classified singularities of lower mobility PKMs into four types by considering the motion/force transmissibility and constrainability. The four types are: (i) input constraint singularity, (ii) output constraint singularity, (iii) input transmission singularity, and (iv) output transmission singularity. Two advantages are offered by this new classification. First, it can identify all possible singularities. Second, it provides a significant physical meaning regarding the transmissibility and constrainability performance of a PKM.

Beyond all the aforementioned types of singularities, there is also so-called control singularity. The control singularity occurs when a single motor actuates two joints (Merlet, 2006a). Finally, it can be seen that all the introduced characterization and classification methods vary in some aspects including the scope of discussion, the analysis method, the basis of classification, the inclusion of passive joints, and the scope of applicability.

Since the performance of a manipulator deteriorates when the manipulator is coincident with or close to the singular configuration, many performance measures have been defined to indicate the distance to singularity. In fact, quite many performance measures are capable to indicate the distance but only a few can specify the type of singularity being faced or identify a more specific type of singularity. Further- more, various techniques including actuation redundancy and providing additional constraints have been developed to deal with singularity problem.

\section{Dexterity measures}

\subsection{Jacobian condition number}

The Jacobian condition number $\kappa_{\mathrm{J}}$ is given by:

$\kappa_{\mathrm{J}}=\|\mathbf{J}\|\left\|\mathbf{J}^{-1}\right\|$

where each double bar bracket indicates a norm.

The Jacobian condition number, often simply called the condition number for short, has a value from 1 to infinity, where infinity indicates singularity. Alternatively, it can also be expressed by its inverse value, called the inverse Jacobian condition number, which has a value from 0 to 1 where 0 indicates singularity. Hence, the Jacobian condition number indicates the distance to the singularity.

The Jacobian condition number indicates how large the error in the task space will be if a small error occurs in the joint space. The more ill-conditioned the Jacobian matrix, the larger the error in the task space will be due to a small error in the joint space. In other words, the Jacobian condition number also indicates the accuracy of a manipulator.

In addition, the Jacobian condition number is a measure of kinematic dexterity (or simply called dexterity for short) and kinematic uniformity (isotropy). The kinematic dexterity is defined as the capability of a manipulator to move the end-effector in all directions with ease. The Jacobian matrix is called isotropic when its condition number or inverse condition number is 1 . At this state, the velocity amplification is identical in all directions, which means that the manipulator can move with the same ease in all directions. However, the Jacobian condition number does not provide a complete information about the dexterity as it only informs how equal the ease in different directions, but not how easy. It is possible that either the motion in all directions requires small effort or the motion in all directions require a large effort. Manipulability which will be reviewed soon provides a more complete information about the kinematic dexterity.

The drawbacks of the Jacobian condition number inherit the dependency problems possessed by the Jacobian matrix as discussed earlier. Furthermore, the Jacobian condition number, as any other condition number of a matrix, is dependent on the choice of norm definition used. The commonly used norms to define the Jacobian condition number are as follows:

- 2-norm, which is defined as the ratio of the maximum and minimum singular values of the Jacobian matrix.

- Frobenius norm, which is given by an analytical function of the manipulator parameters and hence suitable if its gradient is evaluated (Altuzarra et al., 2011), as well 
as it is computationally cheaper since it does not compute the singular values.

- Weighted Frobenius norm, which can be rendered to specific context by adjusting its weights (Khan and Angeles, 2006) in addition to all of the mentioned advantages of the Frobenius norm.

Using the 2-norm definition, the Jacobian condition number can be written as follows:

$\kappa_{\mathrm{J}}=\frac{\sigma_{\max }(\mathbf{J})}{\sigma_{\min }(\mathbf{J})}$

where $\sigma_{\max }(\mathbf{J})$ and $\sigma_{\min }(\mathbf{J})$ denote the maximum and minimum singular values of the Jacobian matrix, respectively.

Using the Frobenius norm definition, the components in Eq. (6) are defined by the following:

$$
\begin{aligned}
& \|\mathbf{J}\|=\sqrt{\operatorname{trace}\left(\mathbf{J J}^{\mathrm{T}}\right)} \\
& \left\|\mathbf{J}^{-1}\right\|=\sqrt{\operatorname{trace}\left(\mathbf{J}^{-1}\left(\mathbf{J}^{-1}\right)^{\mathrm{T}}\right)}
\end{aligned}
$$

When weighted Frobenius norm definition is used, the expressions in Eqs. (8) and (9) are modified to the following:

$$
\begin{aligned}
& \|\mathbf{J}\|=\sqrt{\operatorname{trace}\left(\mathbf{J W} \mathbf{J}^{\mathrm{T}}\right)} \\
& \left\|\mathbf{J}^{-1}\right\|=\sqrt{\operatorname{trace}\left(\mathbf{J}^{-1} \mathbf{W}\left(\mathbf{J}^{-1}\right)^{\mathrm{T}}\right)} \\
& \mathbf{W}=\frac{1}{n} \mathbf{I}
\end{aligned}
$$

where $\mathbf{W}$ is the weighting matrix, $n$ is the number of degrees of freedom, and $\mathbf{I}$ is the identity matrix.

The Jacobian condition number is a local property as it is dependent on the manipulator posture. The global condition number (or global conditioning index, GCI) is defined to represent this property globally. The GCI is obtained by averaging the local condition index (LCI), i.e. the local Jacobian inverse condition number, over the workspace as indicated in Eqs. (1) and (2). Since the Jacobian condition number is commonly used to indicate the dexterity, GCI is also commonly called the global dexterity index (GDI). A map showing the values of LCI across the workspace is commonly called the dexterity map. Different global indices of the Jacobian condition number have also been proposed. Kurtz and Hayward (1992) introduced global gradient index (GGI) defined as the maximum local flatness of the inverse Jacobian condition number in the workspace:

$\mathrm{GGI}=\max \left(\nabla\left(1 / \kappa_{\mathrm{J}}\right)\right)$

Zhixin et al. (2005) introduced global kinematic performance fluctuation index $\sigma$ given by:

$\sigma=\sqrt{\frac{\int_{W}(\mathrm{LCI}-\mathrm{GCI})^{2} \mathrm{~d} W}{\int_{W} \mathrm{~d} W}}$ which represents the kinematic performance volatility in the workspace.

Huang et al. $(2003,2004)$ introduced global comprehensive kinematic performance index $\psi$ given by:

$\psi=\sqrt{\mathrm{GCI}^{2}+(f D)^{2}}$

where $D$ is the ratio of the maximum Jacobian condition number to the minimum one across the workspace whereas $f$ denotes the weight factor of $D$ relative to GCI.

\subsection{Other dexterity measures}

The minimum singular value, another commonly used term for the velocity minimum, is a better indicator of distance to singularity than the manipulability index or the Jacobian condition number (Patel and Sobh, 2015). To make dimensionless the minimum singular value, the relative minimum singular value was defined in Kim and Khosla (1991) as the division of the minimum singular value over a nondimensionalizing factor having identical dimension to the singular values.

To more intuitively represent the physical performance specifications of manipulators, which are typically worstcase, Olds (2015) introduced worst-case velocity index and worst-case error index at a point in the workspace as follows:

$\mu_{\min }=\frac{v_{\mathrm{wc}}}{v_{\mathrm{j}}}=\min _{\|\dot{\theta}\|_{\infty}=1}\|\dot{x}\|=\min _{\|\dot{\theta}\|_{\infty}=1}\|\mathbf{J} \dot{\theta}\|_{2}$

$\mu_{\max }=\frac{e_{\mathrm{wc}}}{e_{\mathrm{j}}}=\max _{\|\dot{\theta}\|_{\infty}=1}\|\dot{x}\|=\max _{\|\dot{\theta}\|_{\infty}=1}\|\mathbf{J} \dot{\theta}\|_{2}$

where $v_{\mathrm{wc}}$ is the worst-case (slowest) end effector velocity, $e_{\mathrm{wc}}$ is the worst-case (largest) end effector positioning error, $v_{\mathrm{j}}$ is the joint velocity magnitude, $e_{\mathrm{j}}$ is the joint error, $\dot{\theta}$ is the joint velocity vector, and $\dot{x}$ is the end effector velocity.

The worst-case velocity index given by Eq. (16) indicates the minimum possible magnitude of the end effector velocity given a unit infinity-norm joint speed input. If the manipulator is not capable of moving in any direction, the worst-case velocity index will be 0 . Therefore, it is expected to have as large worst-case velocity index as possible. Intuitively, the worst-case velocity index can be visualized as the smallest velocity in the manipulability polytope. In similar token, the worst-case error index given by Eq. (17) implies the maximum possible error magnitude of the end effector given a unit infinity-norm joint speed input. It is expected to have as small worst-case error index as possible. Intuitively, the worst-case error index can be visualized as the largest velocity in the manipulability polytope.

Accordingly, Olds (2015) defined a new isotropy index given by:

$l=\frac{\mu_{\min }}{\mu_{\max }}$

The value of $l$ is bounded between 0 and 1 . When the worstcase velocity index is $0, l$ will be zero. On the other hand, if 
the best-case and worst-case velocities are equal, $l$ will be 1 which indicates a full isotropy.

\section{Manipulability}

\subsection{Manipulability as Jacobian-based dexterity and motion/force transmission measure}

Manipulability indicates the quality of velocity and force transmission (amplification). In fact, it provides more information about the velocity and force amplification than the Jacobian matrix condition number. While the latter only informs how isotropic the velocity and force amplification, the former informs both the magnitude and the isotropy of the velocity and force amplification. Therefore, the manipulability serves as both kinematic dexterity and motion/force transmission measure.

Two kinds of manipulability namely velocity (twist) manipulability and force (wrench) manipulability are commonly used. They are commonly represented by velocity manipulability ellipselellipsoid and force manipulability ellipse/ellipsoid, respectively. In the velocity manipulability ellipsoid, the axes lengths indicate the velocity minimum, velocity maximum, and velocity isotropy (Wu et al., 2010; Zhang et al., 2014), whereas the ellipsoid volume indicates the manipulability value. Although the ellipsoid volume provides an information on the magnitude, it does not contain any information on the directionality.

The flattening of the ellipse/ellipsoid or equivalently the ratio of the minimum velocity to the maximum velocity indicates the velocity isotropy. This isotropy represents the kinematic transmission ability in different directions. When the ellipsoid is a sphere or equivalently the ratio is unity, the kinematic transmission ability is uniform in all directions. Furthermore, the velocity and force manipulability are linked by the duality relation between differential kinematics and statics. The major axis of the manipulability ellipsoid indicates the direction along which the manipulator can move easily with the minimum effort, while the minor axis indicates the direction along which the manipulator has the highest stiffness, i.e., the manipulator's actuators can resist forces with minimum effort along this direction.

Beside manipulability ellipse/ellipsoid, manipulability polytope (Lee, 1997), which can include the exact joint constraints, can also be used to represent the manipulability. For six-dimensional manipulator, two separate polytopes can be used to handle the translational and rotational degrees of freedom independently and hence avoid calculation complexity.

The manipulability index $w$ was first introduced by Yoshikawa (1985a) as follows:

$w=\sqrt{\operatorname{det}\left(\mathbf{J J}^{\mathrm{T}}\right)}=\sigma_{1} \sigma_{2} \ldots \sigma_{m}$

where $\sigma_{1}, \sigma_{2}, \ldots, \sigma_{m}$ are the singular values of the Jacobian J.
In the case of square Jacobian, Eq. (19) can be written as:

$w=|\operatorname{det}(\mathbf{J})|$

Alternatively, the manipulability can also be defined by using scaled Jacobian matrix $\tilde{\mathbf{J}}$ as follows (Lee, 1997):

$w_{\mathrm{e}}=\sqrt{\operatorname{det}\left(\tilde{\mathbf{J}} \tilde{\mathbf{J}}^{\mathrm{T}}\right)}=\tilde{\sigma}_{1} \tilde{\sigma}_{2} \ldots \tilde{\sigma}_{m}$

$\tilde{\mathbf{J}}=\mathbf{J R}$

where $\mathbf{R}$ is a diagonal matrix whose diagonal elements are the maximum joint velocity components.

Tanev and Stoyanov (2000) introduced the use of normalized manipulability index which is dimensionless and bounded between 0 and 1 . The normalized manipulability index is given by:

$w_{\mathrm{n}}=\frac{w_{i}}{\max \left(w_{1}, w_{2}, \ldots, w_{\mathrm{n}}\right)}$

where $w_{i}$ denotes the manipulability index at a given point and $\max \left(w_{1}, w_{2}, \ldots, w_{\mathrm{n}}\right)$ denotes the maximum manipulability index in the entire workspace.

The most widely used measure to evaluate the force transmission quality is the force manipulability or sometimes called the payload index (Wu et al., 2010). Similar to the velocity manipulability, it is defined to represent how much unit-norm actuator wrench is amplified to the task space. The force manipulability $w_{\mathrm{f}}$ is defined as follows:

$w_{\mathrm{f}}=\sqrt{\operatorname{det}\left(\mathbf{J}^{-1} \mathbf{J}^{-1 \mathrm{~T}}\right)}$

where $\mathbf{J}^{-1}$ denotes the inverse Jacobian of the manipulator.

Comparing Eq. (24) to the expression of the velocity manipulability in Eq. (19), it can be intuitively understood that the force manipulability is shown to be the best at a posture when the velocity manipulability is the worst, and vice versa. Analogous to the velocity manipulability, the force manipulability can be represented by an ellipse/ellipsoid called force manipulability ellipse/ellipsoid.

The manipulability as given by Eqs. (19)-(24) is a local measure (local manipulability index, LMI) which means that it is dependent on the manipulator posture since it is based on the Jacobian matrix. It can be evaluated globally by using global manipulability index (GMI) which is the local manipulability measure averaged over the workspace as indicated in Eqs. (1) and (2). Another measure is uniformity of manipulability which indicates how uniform the manipulability across the workspace (Pham and Chen, 2004).

\subsection{Improved manipulability indices}

The manipulability indices described earlier, unfortunately, are dependent on order, scale, and dimension. Dependency on the order means that the order is dictated by the number of DOF whereas dependency on the scale means that the value 
is significantly affected by the choice of unit. The earliermentioned manipulability indices are dependent on the dimension since they are based on the Jacobian matrix, and hence face unit inconsistency issue when translation and rotation are mixed.

To overcome the order dependency, Kim and Khosla (1991) introduced order-independent manipulability index $w_{\mathrm{o}}$ given by:

$w_{\mathrm{o}}=\sqrt[n]{w}=\sqrt[n]{\operatorname{det}\left(\mathbf{J J}^{\mathrm{T}}\right)}$

where $n$ is the number of DOF possessed by a manipulator.

Furthermore, they introduced relative manipulability index $w_{\mathrm{r}}$ which is independent from both the order and the scale:

$w_{\mathrm{r}}=\frac{w_{\mathrm{o}}}{f_{\mathrm{M}}}=\frac{w_{\mathrm{o}}}{l^{2}}$

where $f_{\mathrm{M}}$ is a function having the dimension of [length $]^{2}$ and $l$ is the total length of the manipulator.

Doty et al. (1995) proposed weighted twist-manipulability and weighted wrench-manipulability where each of them is defined by incorporating a weighting matrix such that all degrees of freedom (either translational or rotational) are treated uniformly.

To overcome the consistency problem which appears in manipulator mixing translational and rotational DOF, Hong and Kim (2000) introduced a new manipulability measure for PKMs which distinguishes between the translational and rotational components. In this case, they offered four different manipulability measures: translational velocity manipulability, rotational velocity manipulability, force manipulability, and moment manipulability.

Finally, Mansouri and Ouali $(2009,2011)$ introduced homogeneous power manipulability $w_{\mathrm{p}}$ to overcome the unit inconsistency problem. The expression of $w_{\mathrm{p}}$ is not included here since it is lengthy. Unlike manipulability index based on the common Jacobian where the mix of translation and rotation results in unit inconsistency, the utilization of a common quantity between translation and rotation, i.e. power, naturally leads to homogeneous manipulability index. This new measure, similar to the conventional manipulability, is also represented by an ellipse/ellipsoid called power manipulability ellipse/ellipsoid. Furthermore, they introduced three indices to evaluate the manipulability: power manipulability volume $G_{\mathrm{vol}}$, power manipulability isotropy $G_{\mathrm{iso}}$, and minimum power transmissibility $G_{\min }$ :

$$
\begin{aligned}
G_{\mathrm{vol}} & =\frac{1}{\sum_{k=1}^{n} \lambda_{k}} \\
G_{\text {iso }} & =\frac{\lambda_{\min }}{\lambda_{\max }} \\
G_{\text {min }} & =\frac{1}{\lambda_{\max }}
\end{aligned}
$$

where $\lambda_{\min }, \lambda_{\max }$ and $\lambda_{k}$ denote the minimum, maximum, and $k$ th eigenvalues of $w_{\mathrm{p}}$, whereas $n$ is the number of the degrees of freedom of the manipulator. These three indices are local measures and can be extended to their corresponding global measures by utilizing Eqs. (1) and (2).

\section{Force transmission measures}

The force transmission quality includes two kinds of analysis: forward force transmission analysis and inverse force transmission analysis. The earlier attempts to evaluate the bounds of the end effector wrench when the actuator wrench is known, whereas the latter is to determine the bounds of actuator wrench for given end effector wrench. In general, the force transmission measures introduced in the literature can be classified into five broad categories. The first four categories are Jacobian-based, angle-based, screw theory-based, and matrix orthogonal degree-based indices. The first category is represented by the well-known force manipulability, whereas the screw theory-based category is based on the concept of virtual coefficient or power coefficient. The last category is indices recently introduced to consider external load in the transmission evaluation. Since the Jacobian-based transmission measures have been discussed earlier, the following will only discuss the remaining categories.

\subsection{Angle-based force transmission indices}

Pressure angle $\alpha$ and transmission angle $\mu$, first introduced by Alt (1932) and developed by Hain (1967), are formerly defined for gears, cam-follower mechanisms, and planar linkages and later extended for spatial linkages. The pressure angle is defined as the angle between the direction of the driving force and the direction of the velocity of the contact point, as pertaining to the driven element. The transmission angle is the complementary angle to the pressure angle. In general, the pressure angle is expected to be as small as possible whereas, accordingly, the transmission angle is expected to be as large as possible. These angles can also be used to indicate the distance to singularity. Besides, a good transmission angle indicates a good force transmission quality and less sensitivity to mechanical error. A survey on the use of transmission angles in 4, 5, 6 and 7 bar linkages can be found in Balli and Chand (2002). Furthermore, some works have attempted to extend the use of pressure angle or transmission angle to spatial PKMs (Zhang et al., 2011; Zhao et al., 2016). Although these angles have clear physical meaning when used in planar linkages, their definitions become difficult and complex with vague physical meaning when used in complex spatial PKMs with a large number of DOF (Shao et al., 2017).

\subsection{Screw theory-based force transmission indices}

Through the screw theory, Ball (1900) introduced the concept of virtual coefficient. Subsequently, Yuan et al. (1971) utilized the virtual coefficient between the transmission wrench 
screw (TWS) and the output twist screws (OTS) to propose the use of transmission factor having a range between $-\infty$ to $+\infty$ to measure the transmission quality in spatial PKMs. The transmission factor represents the virtual power delivered by the TWS on the OTS. Later, Sutherland and Roth (1973) introduced the transmission index (TI) which is a normalized version of the mentioned transmission factor:

$\mathrm{TI}=|\mathrm{vc}| /|\mathrm{vc}|_{\max }$

where $|v c|$ denotes the virtual coefficient between the TWS and OTS whereas and $|\mathrm{vc}|_{\max }$ denotes the maximum of the virtual coefficient. Notice that Eq. (30) is a general expression of TI whereas the specific expression of the Sutherland and Roth's TI can be found in the mentioned reference. Shimojima and Ogawa (1979) also introduced a normalized TI by proposing a unique definition of TWS, which depends on the output link's load condition.

Later, Tsai and Lee (1994) proposed the total transmission index (TTI) composed of both transmissivity (defined as the ability of a mechanism to generate output) and manipulability (defined as the ability of an input motion to be transmitted into the TWS):

$\mathrm{TTI}=\mathrm{TI} \times \mathrm{MI}$

where TI and MI denote the transmissivity and manipulability indices. Finally, a generalization was made by Chen and Angeles (2007) who proposed the generalized transmission index (GTI) which serves as a generalized form of not only the Sutherland and Roth's TI but also the pressure and transmission angles in their feasible range. Furthermore, they also proposed the transmission quality (TQ) based on the GTI to measure globally the transmission of a mechanism.

Furthermore, Takeda and Funabashi (1995) proposed a definition of TI based on virtual power transmitted from input to output link. In their approach, all input links except one are fixed and the resulting pressure angles are analysed at the connection between the input and output links. They defined the TI of a manipulator with $n$ DOF as:

$\mathrm{TI}=\min \left(\left|\cos \propto_{1}\right|\left|\cos \propto_{2}\right|, \ldots,\left|\cos \propto_{n}\right|\right)$

where $\alpha_{i}$ denotes the pressure angle corresponding to a limb where its input link is driven. The Takeda and Funabashi's TI is dimensionless and bounded between 0 and 1 where 0 indicates singularity. Hence, their TI is also an indicator of distance to singularity. However, the approach only works for TWS with a zero pitch (i.e. a transmission force line) and thus represents a special case of the GTI. In other words, in order to define the pressure angle in a simple definition, the TWS can be represented at a (spherical) joint where no moment is applied as constraint (Brinker et al., 2018). This approach was later extended to spherical PKM (Takeda et al., 1996), spatial 6-DOF PKM (Takeda et al., 1997), and cable driven PKM (Takeda and Funabashi, 2001). Furthermore,
Liu et al. (2014) proposed a novel approach to deal with a case where the TWS and OTS are parallel by exploiting the dual property of the virtual coefficient and accordingly enabling the use two, instead of one, characteristic points. It was shown that the approach provides a more sensitive measure to the platform configuration.

Based on the concept of the virtual coefficient and following Takeda's approach of fixing all inputs except one, Wang et al. $(2010,2017)$ proposed a general procedure for nonredundant spatial parallel manipulators where the force transmission quality is expressed by input and output transmission indices. The input transmission index $\eta_{\text {input }}$ is given by:

$\eta_{\text {input }}=\min \left(\eta_{\mathrm{i}, i}\right)$

where $\eta_{\mathrm{i}, i}$ is defined as the ratio of actual power to potential maximal power of the $i$ th input member. In similar token, the output transmission index is given by:

$\eta_{\text {output }}=\min \left(\eta_{\mathrm{o}, i}\right)$

where $\eta_{\mathrm{o}, i}$ is defined as the ratio of actual power to potential maximal power of the $i$ th output member. Moreover, the force transmission index can be written as:

$\eta=\min \left(\eta_{\text {input }} \eta_{\text {output }}\right)$

and is bounded between 0 and 1 where a larger value indicates better force transmission. It also can be used to indicate the distance to singularity. The indices given by Eqs. (33)(35) are local performance measures. The corresponding global index can be obtained by averaging the indices across the workspace as indicated in Eqs. (1) and (2).

Finally, it should be admitted that the screw theory is a powerful and systematic mathematical tool. However, definition and calculation of the screw theory-based indices are quite complicated, and not intuitive (Shao et al., 2017).

\subsection{Matrix orthogonal degree-based force transmission indices}

Shao et al. (2017) introduced the volume of matrix $\mathbf{X}$ as:

$\operatorname{vol}(\mathbf{X})=\sqrt{\operatorname{det}\left(\mathbf{X}^{\mathrm{T}} \mathbf{X}\right)}$

where matrix $\mathbf{X}$ is composed of $n$ real column vectors, i.e. $\mathbf{X}=\left[\begin{array}{llll}x_{1} & x_{2} & \ldots & x_{n}\end{array}\right]$.

Accordingly, they defined the orthogonal degree of matrix $\mathbf{X}$ as:

$\operatorname{ort}(\mathbf{X})=0 \quad$ if $\min \left\|x_{i}\right\|=0, \quad i=1,2, \ldots, n$
$\operatorname{ort}(\mathbf{X})=\frac{\operatorname{vol}(\mathbf{X})}{\prod_{i=1}^{n}\left\|x_{i}\right\|} \quad$ if $\min \left\|x_{i}\right\| \neq 0, \quad i=1,2, \ldots, n$

They introduced three indices namely joint transmission index (JTI), branch transmission index (BTI), and end-effector 
transmission index (ETI) to indicate the force transmission quality in a single joint, a single branch (limb), and at the end effector, respectively. The JTI of the $i$ th joint located in the $j$ th branch is given by:

$\operatorname{JTI}_{j i}=\sqrt{1-\operatorname{ort}\left(\mathbf{Y}_{j i}\right)^{2}}$

where $\mathbf{Y}_{j i}$ is a two-column matrix containing the afferent and efferent force vectors at the $i$ th joint in the $j$ th branch.

The BTI of the $j$ th branch is defined as the product of all JTIs:

$\mathrm{BTI}_{j}=\mathrm{JTI}_{j 1} \times \operatorname{JTI}_{j 2} \times \ldots \times \operatorname{JTI}_{j k}$

where $k$ denotes the number of joints in the $j$ th branch.

The ETI of a non-redundant manipulator having $n$ DOF is defined as:

$\mathrm{ETI}_{j}=\operatorname{ort}(\mathbf{E F})$

where $\mathbf{E}$ is a diagonal Boolean matrix representing the state, i.e. whether free or constrained, of the manipulator DOF, whereas $\mathbf{F}=\left[\begin{array}{llll}\boldsymbol{\tau}_{1} & \boldsymbol{\tau}_{2} & \ldots & \boldsymbol{\tau}_{n}\end{array}\right]$ is the branch force matrix each column of which is composed of unit force vector and unit torque vector in the $j$ th branch.

Finally, the local total transmission index $\mathrm{TI}_{L}$ of the manipulator is given by the product between the minimum BTI and the ETI:

$\mathrm{TI}_{L}=\min (\mathrm{BTI}) \cdot \mathrm{ETI}$

All of the four indices are bounded between 0 and 1 where 0 indicates the worst transmission whereas 1 represents the best transmission quality. Not only these indices are able to indicate the distance to singularity but also able to distinguish different types of singularities. When BTI is 0 , it means the manipulator is facing an inverse singularity. On the other hand, the manipulator is facing a forward singularity if ETI is 0 . Accordingly, zero $\mathrm{TI}_{L}$ indicates that the manipulator is in a singularity locus whereas unit $\mathrm{TI}_{L}$ indicates that the manipulator is at its best transmission performance.

\subsection{Force transmission indices considering external load}

Lin and Chang (2002) found that the force transmission of a mechanism depends not only on the posture of the mechanism but also on the selection of the output link and the types of the loading. Therefore, the output link should be specified and the external loads should be considered. They proposed the force transmission index (FTI) defined as the effective force ratio (EFR) per unit input torque/force, with a unit torque/force exerted on the output link $i$. The EFR itself is defined as the total power transmitted to the output link via the input-related joints versus the potential maximum power.
The FTI for a single-DOF manipulator is given by:

$\tau_{\mathrm{FTI}}=\left\{\begin{array}{l}\left|R_{i} \eta\right|=\left|R_{i} \frac{T_{\text {out }}}{T_{\text {in }}}\right|=\left|R_{i} \frac{T^{\prime} \text { out }}{T^{\prime} \text { in }}\right| \text { for torque } \\ \left|R_{i} \eta\right|=\left|R_{i} \frac{F_{\text {out }}}{F_{\text {in }}}\right|=\left|R_{i} \frac{F_{\text {out }}^{\prime}}{F_{\text {in }}^{\prime}}\right| \text { for force }\end{array}\right.$

where $R_{i}$ denotes the EFR of the output link $i, \eta$ denotes the mechanical advantage, $T_{\text {in }}\left(F_{\text {in }}\right)$ is the magnitude of the input torque (force) when an arbitrary amount of torque $T_{\text {out }}$ (force $\left.F_{\text {out }}\right)$ is applied to the output link, and $T^{\prime}$ in $\left(F^{\prime}\right.$ in $)$ is the magnitude of input torque (force) when a unit torque $T^{\prime}$ out (a unit force $F^{\prime}$ out) is applied to the output link. Notice that the FTI is dimensionless. It is shown that the FTI provides a more accurate measure of the force transmission quality than the Jacobian-based index.

Subsequently, Chang et al. (2003) extended the concept of FTI to $n$-DOF PKMs with $n$ limbs. In such as case, the total FTI is given by summing the FTI of all of the $n$ limbs:

$\tau_{\mathrm{TFTI}}=\sum_{p=1}^{n} \tau_{\mathrm{FTI}}^{(p)}$

To make it more intuitive, normalized force transmission index (NFTI) was introduced which bounds the index values between zero and one. However, FTI as well as NFTI can only be used for single-DOF manipulators or individual limbs of multi-DOF manipulators. To extend the index to multi-DOF manipulators as a whole, mean force transmission index (MFTI) was introduced by averaging the NFTI values of all limbs in the multi-DOF manipulator. The values range from zero to one. Since MFTI depends on the manipulator posture, a corresponding global index called global force transmission index (GFTI) is defined to characterize the force transmission performance across the workspace.

Furthermore, a new force transmission index for PKMs was introduced by Chen et al. (2015a), rooted in the concepts of pressure angle and transmission angle in a singleDOF manipulator. It evaluates how well a unit-norm wrench applied to the moving platform is transformed to the constraint forces of manipulators, by evaluating the magnitude of force/torque of transmission wrench based on given loads on the end-effector. It overcomes the unit inconsistency and frame-dependency problems. The index $T_{\mathrm{n}}$ is normalized and hence bounded between 0 to 1 as follows:

$T_{\mathrm{n}}=T_{\min } / T$

$T=\sum\left\|F_{i}\right\|^{2}=\sum \frac{1}{\sigma_{i}^{2}}$

where $T$ is the non-normalized force transmission index, $T_{\min }$ is the possible minimum value of $T, F_{i}$ is the $i$ th constraint force vector, and $\sigma_{i}$ is the $i$ th singular value of the transmission matrix. Furthermore, the worst scenario indicated by this index occurs when the magnitude of the transmission wrenches reaches infinity, caused by parallel singularity. 
It should also be mentioned that, after realizing that all previously proposed transmission indices only evaluate the relation between input and output powers, Briot et al. (2013) investigated a complementary method to evaluate the reactions at passive joints due to an external wrench, which are typically high in the singularity neighbourhood and accordingly can cause breakdown of a mechanism. They showed that the increase of reactions at the passive joints depends not only on the transmission angle but also on the position of the instantaneous center of rotation of the platform. To avoid the breakdown, it should be ensured that the maximal reaction forces at the passive joints are always below a certain threshold. However, they developed the complementary method only for planar PKMs and admitted that an extension to spatial PKMs would be challenging.

To this point, a large number of force transmission indices have been proposed. Nevertheless, it is known that both the Jacobian-based force manipulability and the other discussed force transmission measures are intended to handle the static case. To measure dynamic force transmission quality of mechanism, all of the discussed measures should be extended by incorporating the force components appearing in a dynamic case, i.e. inertial force, Coriolis and centrifugal forces, spring force, damping force, and dynamic external force. This might involve defining or evaluating an expected maximum speed and acceleration at which a manipulator will operate. Moreover, the inclusion of friction force in both the static and dynamic cases would give higher fidelity.

\section{Accuracy measures}

Accuracy indicates the ability of the manipulator to give the true position as being commanded. It is very important for precision motion tasks such as machining, measurement, and precision manipulation. It is affected by manufacturing and assembly errors, joint clearance and compliance, link compliance, transmission backlash, computational errors, actuator errors (including finite resolution of the encoders, sensor errors, and control errors), static and dynamic disturbances, thermal effect, etc. Among those error sources, actuator errors are usually the most significant error source (Merlet, 2006b).

Accuracy is often larger than the repeatability (Spong et al., 2004). The accuracy can be predicted theoretically as well as checked by a test. Increasing the accuracy can be done first at the design stage by theoretically optimizing the theoretical accuracy indicators and subsequently by calibration after the machine is built. Furthermore, the inaccuracy which is represented by errors can be evaluated in two senses: relative errors and absolute errors. For the latter one, the maximum absolute errors are usually of utmost interest.

The theoretical accuracy indicators, commonly called accuracy indices, are usually defined to quantify how much the source errors are amplified to the end effector errors. This can be expressed in the following error model:

$\delta \boldsymbol{X}=\mathbf{E} \delta \boldsymbol{\varepsilon}$

where $\delta \boldsymbol{X}$ and $\delta \boldsymbol{\varepsilon}$ denote respectively the vector of end effector errors and the vector of error source, whereas $\mathbf{E}$ transforms the input errors to the end effector errors. It is quite common that the Jacobian matrix is used to represent $\mathbf{E}$. However, Ryu and Cha (2003) suggested the use of total error transformation matrix $\mathbf{E}$ which includes not only the Jacobian matrix, but also other transformation matrices which represent the other error sources.

The theoretical accuracy indicators include the most commonly used Jacobian condition number and worst-case error index as discussed earlier. Furthermore, some more accuracy indices have been introduced.

In similar token to manipulability ellipsoid, error ellipsoid (Ropponen and Arai, 1995) is used to quantify the amplification of source errors to the end-effector errors. This ellipsoid is posture-dependent. Usually, unit-norm source errors are used to define the error ellipsoid. However, Ryu and Cha (2003) used normalized source errors instead of unitnorm source errors. Beside the error ellipsoid, they also suggested the use of maximum singular value and condition number of the error transformation matrix as the accuracy indices. They named these three indices error amplification factors (EAFs). It is a user preference to use any of the three EAFs to evaluate the manipulator accuracy. Notice that they use the total transformation matrix instead of only the Jacobian matrix to define the EAFs. Xu and Li (2008) introduced a weighted mix between the maximum singular value and the condition number and called it error amplification index (EAI). Furthermore, Ryu and Cha (2003) defined the global error amplification factor (GEAF) by averaging the local EAFs across the workspace as indicated in Eqs. (1) and (2).

Moreover, Li and Ye (2003) defined three error sensitivity measures: comprehensive error sensitivity, directional error sensitivity, and absolute error sensitivity. The comprehensive error sensitivity $W$ represents the ratio of the volume of output error to that of input error and is given by:

$W=\left(\operatorname{det}\left(\mathbf{J}_{p}\right)\right)^{-1}$

where $\mathbf{J}_{p}$ is a square, invertible Jacobian matrix of the manipulator.

The directional error sensitivity $C$ represents the isotropy of the error transfer and is given by:

$C=\operatorname{cond}\left(\mathbf{J}_{p}\right)$

Absolute error sensitivity $S$ represents the maximum number of time by which the input error is amplified to form the output error, and is given by:

$S=\left(\sigma_{\min }\left(\mathbf{J}_{p}\right)\right)^{-1}$ 
where $\sigma_{\min }$ is the minimum coefficient by which the input errors are amplified.

Furthermore, Li and Ye (2003) introduced a comprehensive error coefficient e given by:

$e=f_{1} W+f_{2} C+f_{3} S$

where $f_{1}, f_{2}$, and $f_{3}$ are the weights for $W, C$, and $S$, respectively.

However, $W, C, S$, and $e$ represent the errors only for a specific posture. To measure the errors for a group of postures, comprehensive error degree $E$ is used as follows:

$E=\frac{\sum_{i=1}^{N} a_{i} e_{i}}{N}$

where $N$ denotes the number of postures and $a_{i}$ is the weight of the $i$ th posture.

Unfortunately, the aforementioned accuracy measures face homogeneity issue when dealing with mixed DOF. In response to this problem, beyond some treatments to homogenize the Jacobian matrix, several authors proposed the use of exact local maximum position error and local maximum orientation error for given actuator errors $\varepsilon$. Different from the Jacobian condition number which represents the relative error of a manipulator, these recently introduced accuracy measures indicate the absolute error of the manipulator. Furthermore, these new indices represent separately the position and orientation errors and therefore do not face an issue with mixed DOF. To evaluate the indices, either geometric or numerical approach may be conducted. However, the geometric approach is limited to geometrically simple manipulators. For example, Yu et al. (2008) used a geometric approach to measure the accuracy of 3-DOF planar PKMs. When a manipulator has a complex kinematics, a numerical approach through either the forward or inverse kinematics (Liu and Bonev, 2008) can be used to evaluate the indices. Furthermore, to provide more physical insights, Briot and Bonev (2008) introduced another method to evaluate the indices. This method involves solving the direct kinematics for eight, or a maximum of $12 n$ ( $n$ denoting the number of discretization steps) sets of inputs. However, both Yu et al. (2008) and Briot and Bonev (2008) only work within the workspace far from singularities and apply to only 3-DOF planar PKMs. A similar approach to Briot and Bonev (2008) has been applied by Briot and Bonev (2010) to 4-DOF 3T1R PKMs, which involves solving the direct kinematics for 16 , or a maximum of $16 n$ ( $n$ denoting the number of discretization steps) sets of inputs.

Although the local maximum position and orientation errors have clearer physical meaning and do not have homogeneity problem, they require both known input joint nominal values and errors. It means one should quantify both of them prior to the determination of the indices. This, of course, would be an additional effort for a designer. Using the condition number of the error transformation matrix, it is not required to quantify any of them. However, the condition number has homogeneity issue and is easily affected by units and magnitude (Yu et al., 2008). To some extent, it is also affected by the choice of norm definition. In authors' view, both the condition number of the error transformation matrix and the maximum position and orientation errors have their own advantages and disadvantages as mentioned as well as their own specific meanings. The former index indicates how much the input error, regardless of its source, will be amplified in the task space, but not as absolute values, due to its distance to singularity. On the other hand, the latter indices indicate how much exactly, as absolute values, the known input error will be amplified in the task space. In fact, which source of errors is taken into consideration affects the magnitude of the input error. Accordingly, the choice of the index during the design stage depends on the goal and situation. When the DOF are not mixed, the condition number can be a valid yet cheap solution. In the case of mixed DOF, it is a good practice to use both of the homogenized condition number and the maximum position and orientation errors, and subsequently compare the results. In case the results do not align well, the authors suggest to use the latter indices due to its sound and straightforward physical meaning.

Another thing to be considered is comprehensiveness when defining the input error. As described earlier, several authors have introduced some comprehensive accuracy measures which involve not only the Jacobian matrix but also other sources of input joint errors. This comprehensiveness should also be applied when using the maximum position and orientation errors. When quantifying the input joint error, one should not only consider, for example, the resolution of the input joint encoder as the source of error, but also other significant sources of errors.

All of the aforementioned accuracy measures are defined mainly to be used at the design stage, usually for dimensional synthesis. The real-time accuracy observed in a fabricated manipulator is usually represented by tracking error of the end effector. Accordingly, most of the control strategies are based on minimizing the tracking error. In machines where the actuators need to work synchronously to obtain an accurate end effector pose, another accuracy indicator called synchronization error (sometimes also called contouring error) is also commonly used. The tracking error is defined as the distance between the actual position and the desired position, whereas the synchronization error is defined as the shortest distance from the actual position to the desired trajectory (contour) (Lou et al., 2011). The linear combination of the tracking error and the synchronization error is called coupling error. Computing the synchronization error is more involved, particularly in the PKMs, than computing the tracking error. To simplify, the synchronization error can be approximated by the distance of the actual position to the line tangential to the trajectory at the desired position. All of these real-time accuracy measures typically can be optimized 
through a control strategy following the optimization of the manipulator design as well as kinematic calibration.

\section{Stiffness measures}

\subsection{Stiffness modeling}

Stiffness or rigidity of a manipulator structure is important as it affects the accuracy and repeatability of the manipulator. Stiffness is defined as the ability of the manipulator structure to resist deformation caused by wrench. A stiffness matrix defines the relation between deformation vector and wrench vector. It can be derived as the Hessian of potential energy associated to the wrench with respect to the coordinates (Ruggiu, 2012; Quennouelle and Gosselin, 2012). Compliance (flexibility), defined as the inverse of the stiffness, is also often used to indicate the stiffness. Similarly, a compliance matrix is simply the inverse of the stiffness matrix and vice versa. If a structure has high stiffness, it has low compliance. The stiffness can be classified into static stiffness and dynamic stiffness.

The stiffness of a manipulator is dependent on its topology, geometry, material, and control system. The overall stiffness is comprised of the stiffness of the fixed base, the moving platform, the active and passive joints, and the links. The influence of the passive joints on the manipulator stiffness has been studied in some works (Zhang, 2005; Zhang et al., 2009; Pashkevich et al., 2010b, 2011a, b; Klimchik et al., 2012; Sung Kim and Lipkin, 2014). Depending on the significance, the stiffness of the links can be defined in the axial direction (axial stiffness), transversal direction (bending stiffness), or both of them. For simplicity, one or several components of the manipulator are often assumed to be rigid. For example, joints can be considered elastic while the links are assumed to be rigid, or vice versa. A more realistic model can be provided by considering both of the joints and the links as elastic. A trade-off between the simplicity and the fidelity should be made by looking at how significant the compliance of a component contributes to that of the whole manipulator. In a hybrid kinematics machine tool, a PKM is often used for the spindle platform and SKM for the worktable, as the spindle platform is usually the most flexible part of the machine and the use of the PKM is expected to increase its stiffness. Furthermore, some PKMs such as Tricept and Georg V have passive legs to increase their stiffness.

Stiffness is a local property as it is dependent on the manipulator posture. Global stiffness measures are used to evaluate the stiffness globally. Furthermore, stiffness varies with the direction in which it is evaluated as well as the direction of the wrench. The stiffness can be identified in either translational directions, hence called the translational stiffness, or rotational directions, hence called the rotational stiffness. The stiffness can also be evaluated by considering small deflections (quasi-static state) or large deflections (loaded state) (Klimchik et al., 2014).
Several expressions of stiffness have been used, including engineering stiffness, generalized stiffness matrix, and Cartesian stiffness matrix. The engineering stiffness is a onedimensional stiffness expression obtained by evaluating the displacement in the applied force direction (El-Khasawneh and Ferreira, 1999). The generalized stiffness matrix, according to Quennouelle and Gosselin (2012), is comprised of three components namely stiffness of the unconstrained joints, stiffness due to dependent coordinates and internal wrench, and stiffness due to external wrench. The Cartesian stiffness matrix defines the relation between the variation of wrench applied on the end-effector and the variation of the Cartesian displacements (Ruggiu, 2012). It is the most widely used expression of stiffness in manipulator field. Moreover, it is symmetric and either positive definite or positive semi-definite. However, some researchers concluded that the Cartesian stiffness matrix of the elastic structure coupling two rigid bodies is asymmetric in general (Griffis and Duffy, 1993; Ciblak and Lipkin, 1994) and becomes symmetric if the connection is not subjected to any preloading (Žefran and Kumar, 1996, 2002; Howard et al., 1998; Kövecses and Angeles, 2007). Quennouelle and Gosselin (2008) showed that the Cartesian stiffness matrix remains symmetric in the presence of external loadings. Other expressions of Cartesian stiffness matrix were presented by Klimchik (2011), Pashkevich et al. (2011b), and Quennouelle and Gosselin (2012). The latter authors proposed a Cartesian stiffness matrix which can take into account non-zero external loads, non-constant Jacobian matrices, stiff passive joints and additional compliances. Moreover, a direct relation between the Cartesian matrix and the generalized stiffness matrix can be defined by utilizing the Jacobian matrix (Quennouelle and Gosselin, 2008). New representations of Cartesian stiffness matrix based on energetic perspective has been proposed by Metzger et al. (2010).

Furthermore, Zhang and Wei (2014) discussed and compared three stiffness models for PKMs namely traditional stiffness model, kinetostatic compliance model, and dexterous stiffness model. Klimchik et al. (2014) presented an advanced stiffness modeling for PKMs with non-perfect chains under internal and external loadings. The imperfectness occurs as the geometrical parameters differ from the nominal ones and do not allow to assemble manipulator without internal stresses that considerably affect the stiffness properties and also change the end-effector location. Finally, Pashkevich et al. (2010a) presented an enhanced stiffness modeling for parallelogram-based PKMs while taking external loadings into consideration. The stiffness modeling of parallelogram also can be found in $\mathrm{Wu}$ et al. (2016) and $\mathrm{Wu}$ and Zou (2016).

Furthermore, various models have been used to model the manipulator stiffness. The use of the various models can be evaluated from several aspects including the capability to handle complex geometry, ease of use, computational cost, and effectiveness to achieve the goal. In general, the stiffness 
models can be classified into three categories: (1) analytical, continuous model, (2) lumped parameter model, and (3) distributed model. The analytical, continuous stiffness model based on classical beam formulation as well as geometrically exact beam formulation (Jafari and Mahjoob, 2010) have been utilized but limited to simple manipulator geometry. The lumped parameter model based on Jacobian matrix and commonly known as virtual joint method (VJM) model has been widely used in the robotics field. The use of VJM considering only the stiffness of rotational actuators was found in (Salisbury, 1980). Furthermore, a one-dimensional VJM was applied to PKM by Gosselin (1990b), followed by Pashkevich et al. (2009b) who introduced multi-dimensional VJM. More recent works using VJM model include (Ceccarelli and Carbone, 2002; Company et al., 2002; Arumugam et al., 2004; Zhang et al., 2004; Majou et al., 2007; Vertechy and Parenti-Castelli, 2007). This model is commonly used and preferred in robotics since it is analytical and hence requires the same expression for all postures of the manipulator and requires a lower computational cost. It usually still gives an acceptable approximation of the stiffness despite its lower accuracy. For that reason, this model is preferred for the initial estimation of the manipulator stiffness as well as for design optimization purpose.

The most widely used distributed stiffness models are finite element analysis (FEA) and matrix structural analysis (MSA) models, although some other distributed models such as assumed mode method (AMM) and transfer matrix method (TMM) models have also been utilized. As opposed to the lumped parameter model, the FEA model discretizes the manipulator into a number of elements and therefore is closer to the realistic, continuous model. Structural mechanics commonly uses this model due to its high accuracy. It is also able to handle a complex geometry by utilizing a suitable element type. However, it is computationally intensive. Furthermore, it is not practical as it requires new meshing at every different posture of the manipulator. Due to its high accuracy, this model is often used to verify or compare with another less accurate model such as VJM model (ElKhasawneh and Ferreira, 1999; Long et al., 2003; Corradini et al., 2004; Rizk et al., 2006). The MSA model, also known as direct stiffness method, can be considered a special case of FEA model as it uses one-dimensional finite elements instead of two- or three-dimensional ones. Therefore, it gives a trade-off between accuracy and computational cost (Ceccarelli, 2008). Some works using MSA model can be found in Li et al. (2002), Huang et al. (2002), Deblaise et al. (2006) and Soares Junior et al. (2011).

Klimchik et al. (2014) quantitatively compared the computational complexity of VJM, FEA, and MSA stiffness models based on their cost for matrix inversion. They showed that the FEA and VJM models have the highest and lowest complexity, respectively. As an extension of these three models, modifications and improvements on the models have been conducted to alleviate the drawbacks of each of the aforementioned models, such as follows:

- Online FEA by utilizing MSA using generalized springs (Taghvaeipour et al., 2010)

- VJM combined with FEA-based identification technique, which gives high accuracy with low computational cost (Pashkevich et al., 2009a; Klimchik et al., 2013)

- Virtual spring approach which evaluates spring compliance based on FEA concept and therefore gives high accuracy with low computational cost (Pashkevich et al., 2009a, 2011b)

In authors' view, rather than obtaining a lumped parameter model through FEA-based identification as in Pashkevich et al. (2009a) and Klimchik et al. (2013), one can use FEA followed by model order reduction to obtain an accurate yet low-sized stiffness model of a manipulator with a complex shape. This will allow an automated process in obtaining the low-sized stiffness model. Various order reduction methods are available where each offers different accuracy.

As mentioned earlier, the effectiveness to achieve the goal should also be considered to determine a stiffness model to use. Unlike structural mechanics which usually has an interest in the deformation of the whole link, robotics typically only has an interest in the pose at the end effector and possibly joints due to a deformation. In fact, different stiffness models indeed may provide different pose values at those points of interest, and therefore a more accurate model is preferred. Furthermore, when a compliant behavior of manipulator links is considered, the rigid link assumption used for the pose kinematics is no more valid. In such a case, the pose of the end effector should be determined by solving constrained flexible multibody dynamics. Discussion on this topic is beyond the scope of this paper.

Finally, an experiment can be conducted to validate a stiffness model. In this case, the stiffness is obtained from the relation between the measured wrench and measured displacement. Based on a relation function, the stiffness can be obtained by estimation using least squares algorithm or other estimation algorithms.

\subsection{Measures of stiffness magnitude}

In the literature, the magnitude of manipulator stiffness has been presented in the following different ways:

- Graphical representations including stiffness maps, by which the stiffness distribution can be plotted (Gosselin, 1990b; Mekaouche et al., 2015; Zhang et al., 2017), and other graphical representations such as iso-stiffness curves or surfaces (Merlet, 2006a)

- Trace of the stiffness matrix (Carbone and Ceccarelli, 2007) 
- Weighted trace of the stiffness matrix (Gao et al., 2010; Zhang and Gosselin, 2002)

- Minimum, average, or maximum eigenvalues (and eigenvectors) of the stiffness matrix (El-Khasawneh and Ferreira, 1999). For example, the evaluation of minimum and maximum eigenvalues in $\mathrm{Li}$ and $\mathrm{Xu}$ (2008) and Wang et al. (2015) and the average eigenvalue in Ruggiu (2017).

- Mean value of the eigenvalues (Taghvaeipour et al., 2010)

- Determinant of stiffness matrix (Carbone and Ceccarelli, 2007), which is the product of the stiffness matrix eigenvalues, and indicates the area/volume of a stiffness ellipse/ellipsoid. It also indicates the distance from singularity.

- Norm of the stiffness matrix, which can use Euclidian norm, Frobenius norm, or Chebyshev norm (Carbone and Ceccarelli, 2007)

- Center of stiffness or equivalently center of compliance (Patterson and Lipkin, 1993)

- Global compliance index which is given by mean value and deviation of generalized compliance matrix (Xi et al., 2004)

- Virtual work stiffness index which can avoid the problem caused by different units of translation and orientation

- Collinear stiffness value (CSV) (Shneor and Portman, 2010)

- Trace of dexterous stiffness matrix (Zhang and Gao, 2012)

Carbone and Ceccarelli (2007) compared some of the abovementioned stiffness representations from the numerical computation and effectiveness point of view.

\subsection{Measures of stiffness uniformity}

Stiffness condition number is a local property as it is dependent on the manipulator posture. In a similar fashion to the Jacobian condition number, the stiffness condition number $\kappa_{\mathrm{S}}$ is defined by:

$\kappa_{\mathrm{s}}=\left\|\mathbf{K}_{\mathrm{c}}\right\|\left\|\mathbf{K}_{\mathrm{c}}^{-1}\right\|$

Similar to the Jacobian condition number, the stiffness condition number has a value ranging from 1 to infinity. Alternatively, its inverse which has a value ranging from 0 to 1 can also be used. The stiffness condition number indicates the distance of the stiffness matrix $\mathbf{K}_{\mathrm{c}}$ to singularity. It also represents the isotropy or uniformity of the stiffness of any point in the workspace, and therefore stiffness ellipses/ellipsoids are commonly used as the graphical representation. The problem appears when the manipulator mixes translational and rotational DOF and hence the Cartesian stiffness matrix is nonhomogeneous. In this case, the Cartesian stiffness matrix is usually normalized before it is used to compute the stiffness condition number as given in Eq. (52).

Similar to Jacobian condition number, various norm definitions can be used to evaluate the stiffness condition number. The 2-norm, Frobenius norm, and weighted Frobenius norm are commonly used. The considerations in selecting any of them are presented earlier when the Jacobian condition number is discussed.

A global stiffness condition number commonly called the global stiffness index (GSI) is defined as the inverse of the condition number of the stiffness matrix averaged over the workspace as indicated in Eqs. (1) and (2). It indicates the uniformity of stiffness within the whole workspace. Isotropic stiffness workspace is defined as the workspace having stiffness isotropy (Shin et al., 2011).

\subsection{Consistency of stiffness measures}

In manipulators with both translational and rotational DOF, the Cartesian stiffness matrix does not have unit consistency. As a result, evaluation of further stiffness indices such as eigenvalues and condition number of the stiffness matrix becomes controversial. Attempting to overcome this issue, several approaches have been proposed including the following:

- Homogenizing the Jacobian matrix (such as using characteristic length or another technique as discussed in Sect. 3) and subsequently using the homogenized Jacobian matrix to calculate the stiffness matrix ( $\mathrm{Li}$ and $\mathrm{Xu}$, 2006, 2008).

- Eigenscrew decomposition of the stiffness or compliance matrix (Ciblak and Lipkin, 1999; Dai and Ding, 2006; Ding and Selig, 2004; Huang and Schimmels, 2000).

- Principal axis decomposition through congruence transformation by making use of the eigenvectors of the translational entry in the stiffness matrix (Chen et al., 2015)

- Decomposition of the dynamic inertia matrix by transforming variables into dimensionless parameters (Kövecses and Ebrahimi, 2009), which can be applied to the stiffness matrix (Taghvaeipour et al., 2012; Wu, 2014)

- Decoupling of the stiffness matrix into translational and rotational parts (Angeles, 2010; Wu et al., 2016; Wu and Zou, 2016)

It can be seen that the consistency problem in the stiffness measures have been remedied by not only homogenizing the 
Jacobian matrix as the source of the consistency problem, but also by decomposition and decoupling techniques applied directly to the stiffness matrix.

\section{0}

\section{Dynamic performance measures}

Several dynamic performance measures have been used including mass, dynamic dexterity, dynamic manipulability, dynamic stiffness, natural frequencies, and some other dynamic performance measures.

Inertia - The inertia properties include the mass, the first mass moment of inertia, and the second mass moment of inertia (commonly called as mass moment of inertia or simply moment of inertia). These inertial properties are dictated by the material density and the geometry. In lumped parameter model, the mass can be defined by the integration of the density over the volume and the location of the center of mass. The mass and the first moment of inertia are usually constant whereas the second moment of inertia is usually constant with respect to the body frame but varying with respect to the inertial frame. The generalized inertia matrix which represents the inertia of the manipulator as a whole is posture-dependent as it contains elements which vary with the manipulator posture.

Mass, or equivalently weight, is the most intuitive yet significant dynamic performance measure. In fact, it is among the obvious advantages of PKMs over SKMs since the PKMs commonly have their actuators attached to the base and therefore the mobile mass is lower. However, larger reduction of the mass usually results in lower stiffness. An optimization might target to reduce the mass while keeping the stiffness above the required minimum threshold. Further optimization of the mass can be achieved by obtaining optimized detail geometry of the links and moving platform.

Dynamic dexterity - Analogous to the kinematic dexterity, the dynamic dexterity $\kappa_{\mathrm{M}}$ is defined by the condition number of the mass matrix (inertia matrix) $\mathbf{M}$ :

$\kappa_{\mathbf{M}}=\|\mathbf{M}\|\left\|\mathbf{M}^{-1}\right\|$

where the mass matrix is the Hessian matrix of the kinetic energy expression of the manipulator.

The norms in Eq. (53) are commonly defined by 2-norm definition, and hence Eq. (53) can be rewritten as follows:

$\kappa_{\mathrm{M}}=\frac{\sigma_{\max }(\mathbf{M})}{\sigma_{\min }(\mathbf{M})}$

The dynamic dexterity is also a local property since every point at the workspace has its own dynamic dexterity. The global dynamic dexterity index (GDDI) is measured by averaging the local dynamic dexterity over the workspace as indicated in Eqs. (1) and (2).

Furthermore, Asada (1983) introduced generalized inertia ellipsoid which has principal axes along which the inertia tensor is diagonal. The direction of these principal axes is given by the eigenvectors of the generalized inertia tensor, whereas their lengths are given by the reciprocal of the square root of the corresponding eigenvalues. The major axis is corresponding to the smallest eigenvalue which indicates the smallest generalized moment of inertia and consequently the speed is the fastest in that direction. On the other hand, the minor axis is corresponding to the largest eigenvalue which indicates the largest generalized moment of inertia and consequently the speed is slowest in that direction. If the lengths of the principal axes are identical, or equivalently the generalized inertia ellipsoid is a sphere, the resultant inertia is isotropic.

A normalized measure of inertia isotropy $\tilde{\kappa}_{\Lambda}$ bounded between 0 and 1 has been introduced by Kilaru et al. (2015) as follows:

$\tilde{\kappa}_{\Lambda}=\frac{n^{2}}{\kappa(\boldsymbol{\Lambda})}$

where $n$ is the number of degrees of freedom of the manipulator, $\boldsymbol{\Lambda}$ is a diagonal matrix with the eigenvalues of the inertia matrix as the diagonal elements, and $\kappa(\boldsymbol{\Lambda})$ is the condition number of $\boldsymbol{\Lambda}$. Furthermore, they also proposed the use of the maximum eigenvalue of $\boldsymbol{\Lambda}$ as another dynamic performance measure of the manipulator.

Dynamic manipulability - Beyond the commonly used manipulability, dynamic manipulability which was also introduced by Yoshikawa includes not only the Jacobian matrix but also the mass matrix (Merlet, 1996; Yoshikawa, 1985b). The dynamic manipulability $w_{\mathrm{d}}$ is defined by:

$w_{\mathrm{d}}=\sqrt{\operatorname{det}\left(\mathbf{J}\left(\mathbf{M}^{\mathrm{T}} \mathbf{M}\right)^{-1} \mathbf{J}^{\mathrm{T}}\right)}$

For non-redundant manipulators, the dynamic manipulability can be written as:

$w_{\mathrm{d}}=\frac{|\operatorname{det}(\mathbf{J})|}{|\operatorname{det}(\mathbf{M})|}$

Later, a new dynamic manipulability ellipsoid was proposed by Chiacchio (2000). Furthermore, Doty et al. (1995) proposed the use of so-called dynamics frame-acceleration manipulability.

Dynamic stiffness - The dynamic stiffness is a function of the excitation force frequency and depends on the manipulator mass, static stiffness, and damping (Yuan, 2015). Different from the static stiffness which indicates the resistance of the manipulator structure against static loadings, the dynamic stiffness indicates the resistance of the structure against dynamic loadings. The dynamic stiffness can be evaluated by observing the frequency response under excitation force with varying frequency. The lowest dynamic stiffness occurs when the excitation force has the frequency equal to the natural frequency of the manipulator (Alagheband et al., 2014). Azulay et al. (2014) evaluated both of the static stiffness and the dynamic stiffness of a 3PPRS spatial PKM after evaluating the tilting capability and the singularity of the 
manipulator. They modeled the static stiffness of the manipulator by using MSA, defined the dynamic stiffness of the manipulator by considering its mass matrix, damping matrix, and static stiffness matrix, and modeled the dynamic stiffness by using FEM. The dynamic stiffness $\mathbf{K}_{\mathrm{d}}$ formulation they used is given by:

$\mathbf{K}_{\mathrm{d}}=\sqrt{\left(\mathbf{K}-\mathbf{M} \omega^{2}\right)^{2}+(\mathbf{B} \omega)^{2}}$

where $\mathbf{M}, \mathbf{B}$, and $\mathbf{K}$ are the mass matrix, the damping matrix, and the static stiffness matrix of the manipulator, respectively.

Wang (2015) conducted an integrated stiffness analysis of redundant PKM using FEM by considering static stiffness, dynamic stiffness, and moving stiffness of the manipulator. They formulated the dynamic stiffness $\mathbf{K}_{\mathrm{d}}$ based on the following eigenvalue problem:

$\operatorname{det}\left(\mathbf{K}_{\mathrm{d}}-\lambda \mathbf{I}\right)=0$

$\mathbf{K}_{\mathrm{d}}=\mathbf{K}^{-1} \mathbf{M}$

$\lambda=1 / \omega^{2}$

where $\mathbf{K}, \mathbf{M}, \boldsymbol{\omega}$, and $\mathbf{I}$ denote the manipulator stiffness matrix, mass matrix, inherent frequency vector, and identity matrix, respectively. Moreover, they defined the moving stiffness to represent the structure vibration when the manipulator is moving.

Natural frequencies - Natural frequency (also called eigen-frequency) is one of the important dynamic characteristics of a mechanical system, and a manipulator is not an exception. The operation at or near the natural frequencies will result in resonance. The natural frequencies depend on both the inertia and the stiffness. They can be obtained theoretically or experimentally. The former can be conducted either analytically or numerically. In fact, the analytical method is only possible for simple geometries. For example, Germain et al. (2015) presented how the natural frequencies of PKMs are computed by using Euler-Bernoulli beam model. For complex geometries, the theoretical modal analysis should be conducted numerically such as by using FEA.

The natural frequencies are given by solving the following eigenvalue problem:

$\operatorname{det}\left(\omega_{\mathrm{n}}^{2} \mathbf{M}-\mathbf{K}\right)=0$

$\omega_{n}=2 \pi f_{\mathrm{n}}$

where $\mathbf{M}, \mathbf{K}, \omega_{\mathrm{n}}$ and $f_{\mathrm{n}}$ are the mass matrix, the stiffness matrix, the natural frequency in $\operatorname{rad~s}^{-1}$, and the natural frequency in $\mathrm{Hz}$, respectively.

Other dynamic performance measures - In addition to the abovementioned dynamic performance measures, maximum driving (actuation) wrench (Yao et al., 2017), power consumption (Han et al., 2017), maximum speed, and maximum acceleration are among the important dynamic performance measures. In this case, it is always preferred to have a less maximum driving wrench and power consumption, higher maximum speed, and higher acceleration.

\section{Recommendations}

The workspace determination of PKMs is not straightforward as in SKMs. Several approaches have been introduced. The geometrical approach can be easily integrated with CAD system. However, it has a problem with general applicability and constraint handling. Hence, a future work is required to introduce a novel geometric technique which is able to overcome this problem. On the other hand, although the numerical approach has wider applicability and is able to handle the constraints, it is typically slow and less accurate. Therefore, a novel numerical approach which is capable to run faster with good accuracy is required.

Furthermore, a method to present the workspace as an analytical expression is not available for most cases. As a consequence, although most of the local performance measures can be expressed analytically, they cannot be transformed to their global measures by analytical averaging across the workspace indicated in Eq. (1) simply because the workspace cannot be expressed analytically. In such a case, the only way is discrete averaging as indicated in Eq. (2). Therefore, although finding a method to present the workspace analytically with applicability to wider types of PKMs is very challenging, it will be very useful once achieved.

For performance measures which represent the quality of the workspace, the authors have provided some views and suggestions when discussing the measures. In addition, it appears that mixed DOFs result in inconsistency of the indices while many PKMs are required to have mixed DOFs to perform some given tasks. Several attempts have been conducted to define homogeneous indices to overcome the issue. Therefore, it is important to notice that any new index introduced in the future should be able to avoid this issue or overcome it in a more natural way so that the physical insights of the index will be as sound and intuitive as possible. In addition, any new index in the future should be bounded and independent from order, scale (choice of unit), and frame. The formulation complexity, computation performance and cost, physical meaning, information completeness, accurateness, and scope of applicability should also be considered as other criteria. The computation cost, in particular, is very significant when the indices are used in optimization where a large number of iterations are involved. Some indices which involve matrix inversion or eigenvalue computation typically require high computation cost. Therefore, the introduction of cheaper performance indices with sound interpretation as an alternative to the costly ones would be advantageous. Furthermore, considering some special behaviors in a PKM such as over-constraints, redundancy (including kinematic and actuation redundancies), decoupling, joint clearance, compo- 
nent flexibility and nonlinearity (including material and geometric nonlinearities), in the performance measures should also be more discussed in the future.

\section{Conclusion}

This paper provided a comprehensive overview of performance indices of PKMs which include both the workspace and its quality as well as both the kinematic and dynamic performance indices. Not only this paper serves as a comprehensive reference for designers of PKMs in optimizing the performance of their design, but also provided the current state-of-the-art in the research topics and recommended some future research work.

Data availability. No data sets were used in this article.

Author contributions. The manuscript was written through contributions of all authors. AR surveyed the literature and wrote the paper. BEK guided the work and reviewed the manuscript. AA reviewed the manuscript. All authors have given approval to the final version of the manuscript.

Competing interests. The authors declare that they have no conflict of interest.

Acknowledgements. This research was partially supported by the Khalifa University Internal Research Fund.

Review statement. This paper was edited by Marek Wojtyra and reviewed by Calin-Octavian Miclosina and two anonymous referees.

\section{References}

Alagheband, A., Mahmoodi, M., Mills, J. K., and Benhabib, B.: Comparative Analysis of a Redundant Pentapod Parallel Kinematic Machine, J. Mech. Robot., 7, 034502, https://doi.org/10.1115/1.4028933, 2014.

Alciatore, D. G. and Ng, C.-C. D.: Determining manipulator workspace boundaries using the Monte Carlo method and least squares segmentation, ASME Robotics: Kinematics, Dynamics and Controls, 72, 141-146, 1994.

Alt, H.: Der Ubertragungswinkel und seine Bedeutung fur das Konstruieren periodischer Getriebe, Werkstattstechnik, 26, 61-64, 1932.

Altuzarra, O., Salgado, O., Petuya, V., and Hernández, A.: Point-based Jacobian formulation for computational kinematics of manipulators, Mech. Mach. Theory, 41, 1407-1423, https://doi.org/10.1016/j.mechmachtheory.2006.01.011, 2006.
Altuzarra, O., Salgado, O., Petuya, V., and Hernández, A.: Computational kinematics for robotic manipulators: Jacobian problems, Eng. Computation, 25, 4-27, https://doi.org/10.1108/02644400810841396, 2008.

Altuzarra, O., Pinto, C., Sandru, B., and Hernandez, A.: Optimal Dimensioning for Parallel Manipulators: Workspace, Dexterity, and Energy, J. Mech. Design, 133, 041007, https://doi.org/10.1115/1.4003879, 2011.

Angeles, J.: Fundamentals of Robotic Mechanical Systems, Mechanical Engineering Series, Springer, New York, 2002.

Angeles, J.: Is there a characteristic length of a rigidbody displacement?, Mech. Mach. Theory, 41, 884-896, https://doi.org/10.1016/j.mechmachtheory.2006.03.010, 2006.

Angeles, J.: On the Nature of the Cartesian Stiffness Matrix, Ingenierı Mecanica, 3, 163-170, 2010.

Arrouk, K. A., Bouzgarrou, B. C., and Gogu, G.: Workspace Determination and Representation of Planar Parallel Manipulators in a CAD Environment, in: New Trends in Mechanism Science, Springer, the Netherlands, 605-612, 2010.

Arumugam, H. K., Voyles, R. M., and Bapat, S.: Stiffness analysis of a class of parallel mechanisms for micro-positioning applications, IEEE/RSJ International Conference on Intelligent Robots and Systems (IROS), Sendai, 2, 1826-1831, 2004.

Asada, H.: A Geometrical Representation of Manipulator Dynamics and Its Application to Arm Design, J. Dyn. Syst.-T. ASME, 105, 131-142, 1983.

Azulay, H., Mahmoodi, M., Zhao, R., Mills, J. K., and Benhabib, B.: Comparative analysis of a new $3 \times$ PPRS parallel kinematic mechanism, Robot. Cim.-Int. Manuf., 30, 369-378, https://doi.org/10.1016/j.rcim.2013.12.003, 2014.

Baek, J., Iurascu, C.-C., and Park, F. C.: Finding the Maximally Inscribed Rectangle in a Robot's Workspace, KSME Int. J., 15, 1119-1131, 2001.

Bajpai, A. and Roth, B.: Workspace and Mobility of a Closed-Loop Manipulator, Int. J. Robot. Res., 5, 131-142, 1986.

Ball, S. R. S.: A Treatise on the Theory of Screws, Cambridge University Press, Cambridge, 1900.

Balli, S. S. and Chand, S.: Transmission angle in mechanisms (Triangle in mec), Mech. Mach. Theory, 37, 175-195, 2002.

Bohigas, O., Ros, L., and Manubens, M.: A Complete Method for Workspace Boundary Determination on General Structure Manipulators, IEEE T. Robot., 28, 993-1006, 2012.

Bonev, I. A. and Ryu, J.: A new approach to orientation workspace analysis of 6-DOF parallel manipulators, Mech. Mach. Theory, 36, 15-28, 2001.

Brinker, J., Corves, B., and Takeda, Y.: On the Motion/Force Transmissibility and Constrainability of Delta Parallel Robots, in: Computational Kinematics. Mechanisms and Machine Science, edited by: Zeghloul, S., Romdhane, L., and Laribi, M., Springer, Cham, 2018.

Briot, S. and Bonev, I. A.: Accuracy analysis of 3-DOF planar parallel robots, Mech. Mach. Theory, 43, 445-458, https://doi.org/10.1016/j.mechmachtheory.2007.04.002, 2008.

Briot, S. and Bonev, I. A.: Accuracy analysis of $3 \mathrm{~T} 1 \mathrm{R}$ fully-parallel robots, Mech. Mach. Theory, 45, 695-706, https://doi.org/10.1016/j.mechmachtheory.2009.04.002, 2010.

Briot, S., Glazunov, V., and Arakelian, V.: Investigation on the Effort Transmission in Planar Parallel Manipulators, J. Mech. Robot., 5, 011011, https://doi.org/10.1115/1.4023325, 2013. 
Carbone, G. and Ceccarelli, M.: A comparison of indices for stiffness performance evaluation, 12th IFToMM World Congress, 18-21 June 2007, Besançon, France, 2007.

Castelli, G., Ottaviano, E., and Ceccarelli, M.: A Fairly General Algorithm to Evaluate Workspace Characteristics of Serial and Parallel Manipulators\#, Mech. Based Des. Struc., 36, 14-33, https://doi.org/10.1080/15397730701729478, 2008.

Ceccarelli, M.: Stiffness Analysis of Parallel Manipulator Using Matrix Structural Analysis, Proceedings of EUCOMES 08, Springer, Dordrecht, Germany, 2008.

Ceccarelli, M. and Carbone, G.: A stiffness analysis for CaPaMan (Cassino Parallel Manipulator), Mech. Mach. Theory, 37, 427439, 2002.

Chablat, D. and Angeles, J.: On the kinetostatic optimization of revolute-coupled planar manipulators, Mech. Mach. Theory, 37, 351-374, 2002

Chablat, D., Caro, S., Wenger, P., and Angeles, J.: The Isoconditioning Loci of Planar Three-DOF Parallel Manipulators, ASME Design Engineering Technical Conference, Montreal, ASME, Montreal, Canada, 2002.

Chang, W.-T., Lin, C.-C., and Lee, J.-J.: Force Transmissibility Performance of Parallel Manipulators, J. Robotic Syst., 20, 659670, https://doi.org/10.1002/rob.10115, 2003.

Chen, C. and Angeles, J.: Generalized transmission index and transmission quality for spatial linkages, Mech. Mach. Theory, 42, 1225-1237, https://doi.org/10.1016/j.mechmachtheory.2006.08.001, 2007.

Chen, G., Wang, H., Lin, Z., and Lai, X.: The Principal Axes Decomposition of Spatial Stiffness Matrices, IEEE T. Robot., 31, 191-207, 2015.

Chen, X., Chen, C., and Liu, X.-J.: Evaluation of Force/Torque Transmission Quality for Parallel Manipulators, J. Mech. Robot., 7, 041013, https://doi.org/10.1115/1.4029188, 2015a.

Chen, X., Liu, X.-J., and Xie, F.: Screw Theory Based Singularity Analysis of Lower-Mobility Parallel Robots considering the Motion/Force Transmissibility and Constrainability, Math. Probl. Eng., 2015, 1-11, https://doi.org/10.1155/2015/487956, 2015b.

Chiacchio, P.: A new dynamic manipulability ellipsoid for redundant manipulators, Robotica, 18, 381-387, 2000.

Ciblak, N. and Lipkin, H.: Asymmetric Cartesian stiffness for the modelling of compliant robotic systems, 23rd Biennial ASME Mechanisms Conference, Minneapolis, 197-204, ASME, New York, 1994.

Ciblak, N. and Lipkin, H.: Synthesis of Cartesian Stiffness for Robotic Applications, IEEE International Conference of Robotics and Automation, Detroit, Vol. 3, 2147-2152, 1999.

Company, O., Krut, S., and Pierrot, F.: Modelling and preliminary design issues of a 4-axis parallel machine for heavy parts handling, Journal of Multibody Dynamics, 216, 1-11, 2002.

Corradini, C., Fauroux, J. C., Krut, S., and Company, O.: Evaluation of a 4-degree-of-freedom Parallel Manipulator Stiffness, 11th IFToMM World Congress in Mechanism and Machine Science, IFToMM, Tianjin, 1857-1861, 2004.

Dai, J. S. and Ding, X.: Compliance Analysis of a Three-Legged Rigidly-Connected Platform Device, J. Mech. Design, 128, 755, https://doi.org/10.1115/1.2202141, 2006.

Dash, A. K., Chen, I. M., Yeo, S. H., and Yang, G.: Workspace generation and planning singularity-free path for parallel manipulators, Mech. Mach. Theory, 40, 776-805, https://doi.org/10.1016/j.mechmachtheory.2005.01.001, 2005.

Deblaise, D., Hernot, X., and Maurine, P.: Systematic analytical method for PKM stiffness matrix calculation, in: IEEE International Conference on Robotics and Automation (ICRA), Orlando, Florida, May 2006, 4213-4219, 2006.

Ding, X. and Selig, J. M.: On the compliance of coiled springs, Int. J. Mech. Sci., 46, 703-727, 2004.

Doty, K. L., Melchiorri, C., Schwartz, E. M., and Bonivento, C.: Robot Manipulability, IEEE T. Robotic. Autom., 11, 462-468, 1995.

El-Khasawneh, B. S. and Ferreira, P. M.: Computation of stiffness and stiffness bounds for parallel link manipulators, Int. J. Mach. Tool Manu., 39, 321-342, https://doi.org/10.1016/S08906955(98)00039-X, 1999.

Enferadi, J. and Nikrooz, R.: The Performance Indices Optimization of a Symmetrical Fully Spherical Parallel Mechanism for Dimensional Synthesis, J. Intell. Robot. Syst., 90, 305-321, https://doi.org/10.1007/s10846-017-0675-6, 2017.

Fattah, A. and Hasan Ghasemi, A. M.: Isotropic Design of Spatial Parallel Manipulators, Int. J. Robot. Res., 21, 811-824, 2002.

Gao, Z. and Zhang, D.: Workspace Representation and Optimization of a Novel Parallel Mechanism with Three-Degrees-of-Freedom, Sustainability, 3, 2217-2228, https://doi.org/10.3390/su3112217, 2011.

Gao, Z., Zhang, D., and Ge, Y.: Design optimization of a spatial six degree-of-freedom parallel manipulator based on artificial intelligence approaches, Robot. CIM-Int. Manuf., 26, 180-189, https://doi.org/10.1016/j.rcim.2009.07.002, 2010.

Germain, C., Briot, S., Caro, S., and Wenger, P.: Natural Frequency Computation of Parallel Robots, J. Comput. Nonlin. Dyn., 10, 021004, https://doi.org/10.1115/1.4028573, 2015.

Gosselin, C. M.: Determination of the Workspace of 6-DOF Parallel Manipulators, ASME J. Mech. Design, 112, 331-336, 1989.

Gosselin, C. M.: Dexterity Indices for Planar and Spatial Robotic Manipulators, Cincinnati, IEEE Int. Conf. Robot., 1, 650-655, 1990a.

Gosselin, C. M.: Stiffness Mapping for Parallel Manipulators, IEEE T. Robotic. Autom., 6, 377-382, 1990b.

Gosselin, C. M.: The optimum design of robotic manipulators using dexterity indices, J. Robot. Autom. Syst., 9, 213-226, 1992.

Gosselin, C. M. and Angeles, J.: Singularity Analysis of ClosedLoop Kinematic Chains, IEEE T. Robotic. Autom., 6, 281-290, 1990.

Gosselin, C. M. and Jean, M.: Determination of the workspace of planar parallel manipulators with joint limits, Robot. Auton. Syst., 17, 129-138, 1996.

Griffis, M. and Duffy, J.: Global stiffness modeling of a class of simple compliant couplings, Mech. Mach. Theory, 28, 207-224, 1993.

Hain, K.: Applied Kinematics, McGraw-Hill, New York, 1967.

Han, G., Xie, F., and Liu, X.-J.: Evaluation of the power consumption of a high-speed parallel robot, Frontiers of Mechanical Engineering, 13, 167-178, https://doi.org/10.1007/s11465-017-04568, 2017.

Haug, E. J., Luh, C.-M., Adkins, F. A., and Wang, J.-Y.: Numerical algorithms for mapping boundaries of manipulator workspaces, J. Mech. Des.-T. ASME, 118, 228-234, 1995. 
Hong, K.-S. and Kim, J.-G.: Manipulability Analysis of a Parallel Machine Tool: Application to Optimal Link Length Design, J. Robotic Syst., 17, 403-415, 2000.

Hosseini, M. A. and Daniali, H. M.: Weighted local conditioning index of a positioning and orienting parallel manipulator, Sci. Iran., 18, 115-120, https://doi.org/10.1016/j.scient.2011.03.013, 2011.

Hosseini, M. A., Daniali, H.-R. M., and Taghirad, H. D.: Dexterous Workspace Optimization of a Tricept Parallel Manipulator, Adv. Robotics, 25, 1697-1712, https://doi.org/10.1163/016918611x584640, 2011.

Howard, S., Žefran, M., and Kumar, V.: On the $6 \times 6$ Cartesian stiffness matrix for three-dimensional motions, Mech. Mach. Theory, 33, 398-408, 1998.

Huang, S. and Schimmels, J. M.: The Eigenscrew Decomposition of Spatial Stiffness Matrices, IEEE T. Robotic. Autom., 16, 146$156,2000$.

Huang, T., Zhao, X., and Whitehouse, D. J.: Stiffness estimation of a tripod-based parallel kinematic machine, IEEE T. Robotic. Autom., 18, 50-58, 2002.

Huang, T., Li, Z., Li, M., Chetwynd, D. G., and Gosselin, C. M.: Conceptual Design and Dimensional Synthesis of a Novel 2DOF Translational Parallel Robot for Pick-and-Place Operations, J. Mech. Des.-T. ASME, 126, 449-455, 2003.

Huang, T., Li, M., Li, Z., Chetwynd, D. G., and Whitehouse, D. J.: Optimal Kinematic Design of 2-DOF Parallel Manipulators With Well-Shaped Workspace Bounded by a Specified Conditioning Index, IEEE Transactions of Robotics and Automation, 20, 538543, 2004

Huang, Z., Li, Q., and Ding, H.: Theory of Parallel Mechanisms, Mechanism and Machine Science, Vol. 6, Springer, Dordrecht Heidelberg New York London, 2013.

Jafari, M. and Mahjoob, M. J.: An Exact Three-Dimensional Beam Element With Nonuniform Cross Section, J. Appl. Mech., 77, 061009, https://doi.org/10.1115/1.4002000, 2010

Jo, D.-Y. and Haug, E. J.: Workspace Analysis of Multibody Mechanical Systems Using Continuation Methods, J. Mech. Transm.-T. ASME, 111, 581-589, 1989a.

Jo, D.-Y. and Haug, E. J.: Workspace analysis of closed loop mechanisms with unilateral constraints, 17-20 September 1989, Montreal, ASME Des. Aut. Con., 53-60, 1989b.

Kaloorazi, M. H. F., Masouleh, M. T., and Caro, S.: Determining the maximal singularity-free circle or sphere of parallel mechanisms using interval analysis, Robotica, 34, 135-149, https://doi.org/10.1017/s0263574714001271, 2014.

Karger, A.: Self-motions of Stewart-Gough platforms, Comput. Aided Geom. D., 25, 775-783, https://doi.org/10.1016/j.cagd.2008.09.003, 2008.

Karger, A. and Husty, M. L.: Classification of all self-motions of the original Stewart-Gough platform, Comput. Aided Design, 30, 205-215, 1998.

Khan, W. A. and Angeles, J.: The Kinetostatic Optimization of Robotic Manipulators: The Inverse and the Direct Problems, J. Mech. Design, 128, 168-178, 2006.

Kilaru, J., Karnam, M. K., Agarwal, S., and Bandyopadhyay, S.: Optimal design of parallel manipulators based on their dynamic performance, The 14th IFToMM World Congress, 25-30 October 2015, IFToMM, Taipei, Taiwan, 2015.
Kim, D. I., Chung, W. K., and Youm, Y.: Geometrical Approach for the Workspace of 6-DOF Parallel Manipulators, IEEE International Conference on Robotics and Automation (ICRA), Albuquerque, New Mexico, 1997.

Kim, J.-O. and Khosla, P. K.: Dexterity measures for design and control of manipulators, International Workshop on Intelligent Robots and Systems '91, 758-763, IEEE, Osaka, Japan, 1991.

Kim, S.-G. and Ryu, J.: New Dimensionally Homogeneous Jacobian Matrix Formulation by Three End-Effector Points for Optimal Design of Parallel Manipulators, IEEE T. Robotic. Autom. 19, 731-736, 2003.

Klimchik, A.: Enhanced stiffness modeling of serial and parallel manipulators for robotic-based processing of high performance materials , Ecole Centrale de Nantes, Nantes, France, 2011.

Klimchik, A., Pashkevich, A., Caro, S., and Chablat, D.: Stiffness Matrix of Manipulators With Passive Joints: Computational Aspects, IEEE T. Robot., 28, 955-958, 2012.

Klimchik, A., Pashkevich, A., and Chablat, D.: CAD-based approach for identification of elasto-static parameters of robotic manipulators, Finite Elem. Anal. Des., 75, 19-30, https://doi.org/10.1016/j.finel.2013.06.008, 2013.

Klimchik, A., Chablat, D., and Pashkevich, A.: Stiffness modeling for perfect and non-perfect parallel manipulators under internal and external loadings, Mech. Mach. Theory, 79, 1-28, 2014.

Kong, M., Zhang, Y., Du, Z., and Sun, L.: A Novel Approach to Deriving the Unit-Homogeneous Jacobian Matrices of Mechanisms, IEEE International Conference of Mechatronics and Automation, 3051-3055, Harbin, Heilongjiang, China, 2007.

Kövecses, J. and Angeles, J.: The stiffness matrix in elastically articulated rigid-body systems, Multibody Syst. Dyn., 18, 169184, 2007.

Kövecses, J. Z. and Ebrahimi, S.: Parameter Analysis and Normalization for the Dynamics and Design of Multibody Systems, J. Comput. Nonlin. Dyn., 4, 031008 , https://doi.org/10.1115/1.3124785, 2009.

Kumar, V.: Characterization of Workspaces of Parallel Manipulators, J. Mech. Des.-T. ASME, 114, 368-375, 1992.

Kurtz, R. and Hayward, V.: Multiple-goal kinematic optimization of a parallel spherical mechanism with actuator redundancy, IEEE T. Robotic. Autom., 8, 644-651, 1992.

Lee, J.: A Study on the Manipulability Measures for Robot Manipulators, International Conference on Intelligent Robots and Systems (IROS), Grenoble, France, 1997.

Lee, J.-H., Nam, Y.-J., and Park, M.-K.: Kinematics and optimization of a 2-DOF parallel manipulator with a passive constraining leg and linear actuators, J. Mech. Sci. Technol., 24, 19-23, https://doi.org/10.1007/s12206-009-1114-9, 2010.

Li, T. and Ye, P.: The Measurement of Kinematic Accuracy for Various Configurations of Parallel Manipulators, IEEE International Conference on Systems, Man and Cybernetics, 8 October 2003, Washington, DC, USA, 2003.

Li, Y. and Xu, Q.: GA-Based Multi-Objective Optimal Design of a Planar 3-DOF Cable-Driven Parallel Manipulator, IEEE International Conference on Robotics and Biomimetics, 17-20 December 2006, Kunming, China, 2006.

$\mathrm{Li}, \mathrm{Y}$. and $\mathrm{Xu}, \mathrm{Q} .:$ Stiffness analysis for a 3-PUU parallel kinematic machine, Mech. Mach. Theory, 43, 186-200, https://doi.org/10.1016/j.mechmachtheory.2007.02.002, 2008. 
Li, Y. W., Wang, J. S., and Wang, L. P.: Stiffness analysis of a Stewart platform-based parallel kinematic machine, 3672-3677, IEEE International Conference on Robotics and Automation (ICRA), Washington, 2002.

Lin, C. C. and Chang, W. T.: The force transmissivity index of planar linkage mechanisms, Mech. Mach. Theory, 37, 1465-1485, 2002.

Lipkin, H. and Duffy, J.: Hybrid twist and wrench control for a robotic manipulator, J. Mech. Transm.-T. ASME, 110, 138-144, 1988.

Liu, G., Lou, Y., and Li, Z.: Singularities of Parallel Manipulators A Geometric Treatment, Transactions on Robotics and Automation, 19, 579-594, 2003.

Liu, H., Huang, T., and Chetwynd, D. G.: A Method to Formulate a Dimensionally Homogeneous Jacobian of Parallel Manipulators, IEEE T. Robot., 27, 150-156, https://doi.org/10.1109/tro.2010.2082091, 2011.

Liu, H., Huang, T., Kecskeméthy, A., and Chetwynd, D. G.: A generalized approach for computing the transmission index of parallel mechanisms, Mech. Mach. Theory, 74, 245-256, https://doi.org/10.1016/j.mechmachtheory.2013.12.012, 2014.

Liu, X.-J. and Bonev, I. A.: Orientation Capability, Error Analysis, and Dimensional Optimization of Two Articulated Tool Heads With Parallel Kinematics, J. Manuf. Sci. E.-T. ASME, 130, 011015, https://doi.org/10.1115/1.2783255, 2008.

Long, C. S., Snyman, J. A., and Groenwold, A. A.: Optimal structural design of a planar parallel platform for machining, Appl. Math. Model., 27, 581-609, 2003.

Lou, Y., Li, Z., Zhong, Y., Li, J., and Li, Z.: Dynamics and contouring control of a 3-DoF parallel kinematics machine, Mechatronics, 21, 215-226, https://doi.org/10.1016/j.mechatronics.2010.10.007, 2011.

Lou, Y., Zhang, Y., Huang, R., Chen, X., and Li, Z.: Optimization Algorithms for Kinematically Optimal Design of Parallel Manipulators, IEEE T. Autom. Sci. Eng., 11, 574-584, 2014.

Lou, Y. J., Liu, G. F., and Li, Z. X.: A General Approach for Optimal Design of Parallel Manipulators, IEEE International Conference of Robotics and Automation (ICRA), 26 April-1 May 2004, New Orleans, LA, USA, USA, 2004.

Ma, O. and Angeles, J.: Architecture singularities of platform manipulators, IEEE Int. Conf. Robot., 911 April 1991, Sacramento, CA, USA, 1542-1547, https://doi.org/10.1109/robot.1991.131835, 1991a.

Ma, O. and Angeles, J.: Optimum Architecture Design of Platform Manipulators, IEEE International Conference on Advanced Robotics, 19-22 June 1991, Pisa, 1991b.

Majou, F., Gosselin, C., Wenger, P., and Chablat, D.: Parametric stiffness analysis of the Orthoglide, Mech. Mach. Theory, 42, 296-311, 2007.

Mansouri, I. and Ouali, M.: A new homogeneous manipulability measure of robot manipulators, based on power concept, Mechatronics, 19, 927-944, https://doi.org/10.1016/j.mechatronics.2009.06.008, 2009.

Mansouri, I. and Ouali, M.: The power manipulability - A new homogeneous performance index of robot manipulators, Robot. CIM-Int. Manuf., 27, 434-449, https://doi.org/10.1016/j.rcim.2010.09.004, 2011.

Mekaouche, A., Chapelle, F., and Balandraud, X.: FEM-Based Generation of Stiffness Maps, IEEE T. Robot., 31, 217-222, 2015.
Merlet, J.-P.: Determination of the Orientation Workspace of Parallel Manipulators, Journal of Intelligent and Robotic System, 13, 143-160, 1995.

Merlet, J.-P.: Redundant Parallel Manipulators, Lab Robotic Automation, 8, 17-24, 1996.

Merlet, J.-P.: Parallel Robots, 2nd Edn., Springer, Dordrecht, 2006a.

Merlet, J.-P.: Computing the worst case accuracy of a PKM over a workspace or a trajectory, The Fifth Chemnitz Parallel Kinematics Seminar, Chemnitz, Germany, 2006b.

Merlet, J. P.: Jacobian, Manipulability, Condition Number, and Accuracy of Parallel Robots, J. Mech. Design, 128, 199-206, https://doi.org/10.1115/1.2121740, 2006c.

Merlet, J.-P.: Singular Configurations of Parallel Manipulators and Grassmann Geometry, Int. J. Robot. Res., 8, 45-56, https://doi.org/10.1177/027836498900800504, 2016.

Merlet, J.-P., Gosselin, C. M., and Mouly, N.: Workspaces of Planar Parallel Manipulators, Mech. Mach. Theory, 33, 7-20, 1998.

Metzger, M. F., Faruk Senan, N. A., and O'Reilly, O. M.: On Cartesian stiffness matrices in rigid body dynamics: an energetic perspective, Multibody Syst. Dyn., 24, 441-472, https://doi.org/10.1007/s11044-010-9205-z, 2010.

Nurahmi, L. and Caro, S.: Dimensionally Homogeneous Jacobian and Condition Number, Applied Mech. Mater., 836, 42-47, https://doi.org/10.4028/www.scientific.net/AMM.836.42, 2016.

Olds, K. C.: Global Indices for Kinematic and Force Transmission Performance in Parallel Robots, IEEE T. Robot., 31, 494-500, 2015.

Park, F. C. and Kim, J. W.: Singularity Analysis of Closed Kinematic Chains, J. Mech. Design, 121, 32-38, 1999.

Pashkevich, A., Chablat, D., and Wenger, P.: Stiffness analysis of overconstrained parallel manipulators, Mech. Mach. Theory, 44, 966-982, https://doi.org/10.1016/j.mechmachtheory.2008.05.017, 2009a.

Pashkevich, A., Chablat, D., and Wenger, P.: Stiffness Analysis of Multi-Chain Parallel Robotic Systems, Journal of Automation, Mobile Robotics and Intelligent Systems, 3, 75-82, 2009b.

Pashkevich, A., Klimchik, A., Caro, S., and Chablat, D.: Stiffness Modelling of Parallelogram-Based Parallel Manipulators, in: New Trends in Mechanism Science, edited by: Pisla, D., Ceccarelli, M., Husty, M., and Corves, B., Mechanisms and Machine Science, Vol. 5, 675-682, Springer, Dordrecht, https://doi.org/10.1007/978-90-481-9689-0_77, 2010.

Pashkevich, A., Klimchik, A., and Chablat, D.: Stiffness Analysis of Parallel Manipulators with Preloaded Passive Joints, 12th International Symposium on Advances in Robot Kinematics, Slovenie, France, 2010b.

Pashkevich, A., Klimchik, A., Caro, S., and Chablat, D.: Cartesian stiffness matrix of manipulators with passive joints: Analytical approach, IEEE/RSJ International Conference on Intelligent Robots and Systems (IROS), 25-30 September 2011, San Francisco, CA, USA, 2011a.

Pashkevich, A., Klimchik, A., and Chablat, D.: Enhanced stiffness modeling of manipulators with passive joints, Mech. Mach. Theory, 46, 662-679, https://doi.org/10.1016/j.mechmachtheory.2010.12.008, $2011 \mathrm{~b}$.

Patel, S. and Sobh, T.: Manipulator Performance Measures - A Comprehensive Literature Survey, J. Intell. Robot. Syst., 77, 547-570, https://doi.org/10.1007/s10846-014-0024-y, 2015. 
Patterson, T. and Lipkin, H.: A Classification of Robot Compliance, J. Mech. Des.-T. ASME, 115, 581-584, 1993.

Pham, H. H. and Chen, I.-M.: Optimal synthesis for workspace and manipulability of parallel flexure mechanism, 11th World Congress on Theory of Machines and Mechanisms, 1-4 April 2004, Tianjin, 2004.

Pond, G. and Carretero, J. A.: Formulating Jacobian matrices for the dexterity analysis of parallel manipulators, Mech. Mach. Theory, 41, 1505-1519, https://doi.org/10.1016/j.mechmachtheory.2006.01.003, 2006.

Pond, G. and Carretero, J. A.: Quantitative dexterous workspace comparison of parallel manipulators, Mech. Mach. Theory, 42, 1388-1400, https://doi.org/10.1016/j.mechmachtheory.2006.10.004, 2007.

Quennouelle, C. and Gosselin, C. M.: Stiffness Matrix of Compliant Parallel Mechanisms, in: Advances in Robot Kinematics: Analysis and Design, Springer, Dordrecht, 331-341, 2008.

Quennouelle, C. and Gosselin, C. M.: A General Formulation for the Stiffness Matrix of Parallel Mechanisms, ArXiv, abs/1212.0950, 2012.

Ranjbaran, F., Angeles, J., Gonzalez-Palacios, M. A., and Patel, R. V.: The Mechanical Design of a Seven-Axes Manipulator with Kinematic Isotropy, J. Intell. Robot. Syst., 14, 21-41, 1995.

Rastegar, J. and Perel, D.: Generation of Manipulator Workspace Boundary Geometry Using the Monte Carlo Method and Interactive Computer Graphics, ASME Trends and Developments in Mechanisms, Machines, and Robotics, 3, 299-305, 1990.

Rizk, R., Fauroux, J. C., Mumteanu, M., and Gogu, G.: A comparative stiffness analysis of a reconfigurable parallel machine with three or four degrees of mobility, J. Mach. Eng., 6, 45-55 2006.

Ropponen, T. and Arai, T.: Accuracy Analysis of a Modified Stewart Platform Manipulator, IEEE International Conference on Robotics and Automation (ICRA), 21-27 May 1995, Nagoya, Japan, 1995.

Rosyid, A., El-Khasawneh, B. S., and Alazzam, A.: Kinematic Performance Measures and Optimization of Parallel Kinematics Manipulators: A Brief Review, Kinematics, edited by: Gorrostieta-Hurtado, E., InTech, London, https://doi.org/10.5772/intechopen.71406, 2017.

Ruggiu, M.: On the Lagrangian and Cartesian Stiffness Matrices of Parallel Mechanisms with Elastic Joints, Adv. Robotics, 26, 137-153, https://doi.org/10.1163/016918611x607680, 2012.

Ruggiu, M.: Cartesian Stiffness Matrix Mapping of a Translational Parallel Mechanism with Elastic Joints, Int. J. Adv. Robot. Syst., 9, 195, https://doi.org/10.5772/52145, 2017.

Ryu, J. and Cha, J.: Volumetric error analysis and architecture optimization for accuracy of HexaSlide type parallel manipulators, Mech. Mach. Theory, 38, 227-240, 2003.

Salisbury, J.: Active Stiffness Control of a Manipulator in Cartesian Coordinates, 19th IEEE Conference on Decision and Control, 10-12 December 1980, Albuquerque, NM, USA, 1980.

Saputra, V. B., Ong, S. K., and Nee, A. Y. C.: A swarm optimization approach for solving workspace determination of parallel manipulators, Robotica, 33, 649-668, https://doi.org/10.1017/s0263574714000290, 2014.

Shao, Z.-F., Mo, J., Tang, X.-Q., and Wang, L.-P.: Transmission Index Research of Parallel Manipulators Based on Matrix Orthogonal Degree, Chin. J. Mech. Eng., 30, 1396-1405, https://doi.org/10.1007/s10033-017-0193-2, 2017.
Shijun, Y.: A Serial-Parallel Hybrid Robot for Machining of Complex Surfaces, National University of Singapore, Singapore, 2015.

Shimojima, H. and Ogawa, K.: A Transmissiblity for Single-Loop Spatial Mechanisms, B. JSME, 22, 405-411, 1979.

Shin, H., Lee, S., Jeong, J. I., and Kim, J.: Kinematic Optimization for Isotropic Stiffness of Redundantly Actuated Parallel Manipulators, IEEE International Conference on Robotics and Automation, 9-13 May 2011, Shanghai, China, 2011.

Shneor, Y. and Portman, V. T.: Stiffness of 5-axis machines with serial, parallel, and hybrid kinematics: Evaluation and comparison, CIRP Ann.-Manuf. Techn., 59, 409-412, https://doi.org/10.1016/j.cirp.2010.03.020, 2010.

Snyman, J. A., Plessis, L. J. D., and Duffy, J.: An Optimization Approach to the Determination of the Boundaries of Manipulator Workspaces, J. Mech. Des.-T. ASME, 122, 447-456, 2000.

Soares Junior, G. D. L., Carvalho, J. C. M., and Goncalves, R. S.: Stiffness Analysis of 6-RSS Parallel Manipulator, 13th World Congress in Mechanism and Machine Science, 19-25 June 2011, Guanajuato, México, 2011.

Spong, M. W., Hutchinson, S., and Vidyasagar, M.: Robot Dynamics and Control, 2nd Edn., available at: https://home.deib.polimi it/gini/robot/docs/spong.pdf (last access: 5 March 2020), 2004.

Stocco, L., Salcudean, S. E., and Sassani, F.: Matrix Normalization for Optimal Robot Design, IEEE International Conference on Robotics and Automation, 16-21 May 1998, Leuven, Belgium, 1998.

Stocco, L. J., Saculdean, S. E., and Sassani, F.: On the use of scaling matrices for task-specific robot design, IEEE T. Robotic. Autom., 15, 958-965, 1999.

Sung Kim, H. and Lipkin, H.: Stiffness of Parallel Manipulators With Serially Connected Legs, J. Mech. Robot., 6, 031001, https://doi.org/10.1115/1.4026333, 2014.

Sutherland, G. and Roth, B.: A Transmission Index for Spatial Mechanisms, J. Eng. Ind., 95, 589-597, 1973.

Taghvaeipour, A., Angeles, J., and Lessard, L.: Online Computation of the Stiffness Matrix in Robotic Structures Using Finite Element Analysis, Department of Mechanical Engineering and Centre for Intelligent Machines, McGill University, Montreal, Quebec, Canada, 2010.

Taghvaeipour, A., Angeles, J., and Lessard, L.: On the elastostatic analysis of mechanical systems, Mech. Mach. Theory, 58, 202216, https://doi.org/10.1016/j.mechmachtheory.2012.07.011, 2012.

Takeda, Y. and Funabashi, H.: Motion Transmissibility of InParallel Actuated Manipulators, JSME Int. J. C-Dyn. Con., 38, 749-755, 1995.

Takeda, Y. and Funabashi, H.: A Transmission Index for In-Parallel Wire-Driven Mechanisms, JSME Int. J. C-Mech. Sy., 44, 180187, 2001.

Takeda, Y., Funabashi, H., and Sasaki, Y.: Development of a Spherical In-Parallel Actuated Mechanism with Three Degrees of Freedom with Large Working Space and High Motion Transmissibility: Evaluation of Motion Transmissibility and Analysis of Working Space, JSME Int. J. C-Dyn. Con., 39, 541-548, 1996.

Takeda, Y., Funabashi, H., and Ichimaru, H.: Development of Spatial In-Parallel Actuated Manipulators with Six Degrees of Freedom with High Motion Transmissibility, JSME Int. J. C-Mech. Sy., 40, 299-308, 1997. 
Tandirci, M., Angeles, J., and Ranjbaran, F.: The Characterstic Point and the Characteristic Length of Robotic Manipulators, 203-208, ASME 22nd Biennial Conference on Robotics, Spatial Mechanisms, and Mechanical Systems, Scotsdale, Arizona, 1992.

Tanev, T. and Stoyanov, B.: On the Performance Indexes for Robot Manipulators, Problems of Engineering Cybernetics and Robotics, 49, 64-71, 2000.

Tsai, M. J. and Lee, H. W.: The Transmissivity and Manipulability of Spatial Mechanisms, J. Mech. Design, 116, 137-143, 1994.

Vertechy, R. and Parenti-Castelli, V.: Static and stiffness analyses of a class of over-constrained parallel manipulators with legs of type US and UPS, 561-567, IEEE International Conference on Robotics and Automation (ICRA), Rome, 2007.

Wang, D., Fan, R., and Chen, W.: Performance enhancement of a three-degree-of-freedom parallel tool head via actuation redundancy, Mech. Mach. Theory, 71, 142-162, https://doi.org/10.1016/j.mechmachtheory.2013.09.006, 2014.

Wang, J., Wu, C., and Liu, X.-J.: Performance evaluation of parallel manipulators: Motion/force transmissibility and its index, Mech. Mach. Theory, 45, 1462-1476, https://doi.org/10.1016/j.mechmachtheory.2010.05.001, 2010.

Wang, L., Xu, H., and Guan, L.: Optimal design of a 3-PUU parallel mechanism with 2R1T DOFs, Mech. Mach. Theory, 114, 190203, https://doi.org/10.1016/j.mechmachtheory.2017.03.008, 2017.

Wang, M., Carbone, G., and Ceccarelli, M.: Stiffness Analysis for a Tripod Leg Mechanism, The 14th IFToMM World Congress, 25-30 October 2015, Taipei, Taiwan, 2015.

Wang, S.: Integrated Stiffness Analysis of Redundant Parallel Manipulator Based on Finite Element Method, Journal of Information and Computational Science, 12, 351-365, https://doi.org/10.12733/jics20105539, 2015.

Wang, Z., Wang, Z., Liu, W., and Lei, Y.: A study on workspace, boundary workspace analysis, and workpiece positioning for parallel machine tools, Mech. Mach. Theory, 36, 605-622, 2001.

Wohlhart, K.: Mobile 6-SPS Parallel Manipulators, J. Robotic Syst., 20, 509-516, https://doi.org/10.1002/rob.10101, 2003.

Wohlhart, K.: From higher degrees of shakiness to mobility, Mech. Mach. Theory, 45, 467-476, https://doi.org/10.1016/j.mechmachtheory.2009.10.006, 2010.

Wu, G.: Stiffness Analysis and Optimization of a Co-axial Spherical Parallel Manipulator, Modeling, Identification and Control: A Norwegian Research Bulletin, 35, 21-30, https://doi.org/10.4173/mic.2014.1.2, 2014.

$\mathrm{Wu}$, G. and Zou, P.: Stiffness analysis and comparison of a Biglide parallel grinder with alternative spatial modular parallelograms, Robotica, 35, 1310-1326, https://doi.org/10.1017/s0263574716000059, 2016.

Wu, G., Bai, S., and Hjornet, P.: On the Stiffness of Three or Four DOF Parallel Pick-and-place Robots with Four Identical Limbs, IEEE International Conference on Robotics and Automation (ICRA), 16-24 May 2016, Stockholm, Sweden, 2016.

Wu, J., Wang, J., Wang, L., and You, Z.: Performance comparison of three planar 3-DOF parallel manipulators with 4RRR, 3-RRR and 2-RRR structures, Mechatronics, 20, 510-517, https://doi.org/10.1016/j.mechatronics.2010.04.012, 2010.

Xi, F., Zhang, D., Mechefske, C. M., and Lang, S. Y. T.: Global kinetostatic modelling of tripod-based paral- lel kinematic machine, Mech. Mach. Theory, 39, 357-377, https://doi.org/10.1016/j.mechmachtheory.2003.09.007, 2004.

$\mathrm{Xu}, \mathrm{Q}$. and $\mathrm{Li}, \mathrm{Y}$.: Error analysis and optimal design of a class of translational parallel kinematic machine using particle swarm optimization, Robotica, 27, 67, https://doi.org/10.1017/s0263574708004530, 2008.

Yao, J., Gu, W., Feng, Z., Chen, L., Xu, Y., and Zhao, Y.: Dynamic analysis and driving force optimization of a 5-DOF parallel manipulator with redundant actuation, Robot. CIM-Int. Manuf., 48, 51-58, https://doi.org/10.1016/j.rcim.2017.02.006, 2017.

Yoshikawa, T.: Manipulability of robot mechanisms, Int. J. Robot. Res., 4, 3-9, 1985a.

Yoshikawa, T.: Dynamic Manipulability of Robot Manipulators, J. Robotic Syst., 2, 113-124, 1985b.

Yu, A., Bonev, I. A., and Zsombor-Murray, P.: Geometric approach to the accuracy analysis of a class of 3-DOF planar parallel robots, Mech. Mach. Theory, 43, 364-375, https://doi.org/10.1016/j.mechmachtheory.2007.03.002, 2008.

Yuan, H.: Static and Dynamic Stiffness Analysis of Cable-Driven Parallel Robots, Institut National des Sciences Appliquees de Rennes, CEDEX, 2015.

Yuan, M. S. C., Freudenstein, F., and Woo, L. S.: Kinematic Analysis of Spatial Mechanisms by Means of Screw Coordinates. Part 2 - Analysis of Spatial Mechanisms, J. Eng. Ind., 93, 67-73, 1971.

Yuefa, F. and Tsai, L.-W.: Structure Synthesis of a Class of 4-DoF and 5-DoF Parallel Manipulators with Identical Limb Structures, Int. J. Robot. Res., 21, 799-810, https://doi.org/10.1177/0278364902021009314, 2016.

Žefran, M. and Kumar, V.: Coordinate-free formulation of the Cartesian stiffness matrix, 22-26, 5th Int. Symposium on Advances in Robot Kinematics, Portorôz, 1996.

Žefran, M. and Kumar, V.: A geometrical approach to the study of the Cartesian stiffness matrix, J. Mech. Design, 124, 30-38, 2002.

Zhang, D.: On stiffness improvement of the Tricept machine tool, Robotica, 23, 377-386, https://doi.org/10.1017/s0263574704000992, 2005.

Zhang, D. and Gao, Z.: Forward kinematics, performance analysis, and multi-objective optimization of a bio-inspired parallel manipulator, Robot. CIM-Int. Manuf., 28, 484-492, https://doi.org/10.1016/j.rcim.2012.01.003, 2012.

Zhang, D. and Gosselin, C. M. M.: Kinetostatic Analysis and Design Optimization of the Tricept Machine Tool Family, J. Manuf. Sci. E.-T. ASME, 124, 725, https://doi.org/10.1115/1.1471529, 2002.

Zhang, D. and Wei, B.: Comparison and Verification of Several Stiffness Models for a Family of Parallel Manipulators, International Conference of Control, Dynamic Systems, and Robotics, Ottawa, Ontario, Canada, 2014.

Zhang, D., Xi, F., Mechefske, C. M., and Lang, S. Y. T.: Analysis of parallel kinematic machine with kinetostatic modelling method, Robot. CIM-Int. Manuf., 20, 151-165, 2004.

Zhang, D., Bi, Z., and Li, B.: Design and kinetostatic analysis of a new parallel manipulator, Robot. CIM-Int. Manuf., 25, 782-791, https://doi.org/10.1016/j.rcim.2008.10.002, 2009.

Zhang, D., Xu, Y., Yao, J., Hu, B., and Zhao, Y.: Kinematics, dynamics and stiffness analysis of a novel 3DOF kinematically/actuation redundant planar paral- 
lel mechanism, Mech. Mach. Theory, 116, 203-219, https://doi.org/10.1016/j.mechmachtheory.2017.04.011, 2017.

Zhang, L., Mei, J., Zhao, X., and Huang, T.: Dimensional synthesis of the Delta robot using transmission angle constraintsDimensional synthesis of the Delta robot using transmission angle constraints, Robotica, 30, 343-349, https://doi.org/10.1017/s0263574711000622, 2011.

Zhang, P., Yao, Z., and Du, Z.: Global Performance Index System for Kinematic Optimization of Robotic Mechanism, J. Mech. Design, 136, 031001, https://doi.org/10.1115/1.4026031, 2014.

Zhao, Q., Mei, J., Song, T., and Liu, S.: Pressure angle in parallel mechanisms: From planar to spatial, Transactions of Tianjin University, 22, 411-418, https://doi.org/10.1007/s12209-016-28874, 2016.
Zhixin, S., Yufeng, L., and Hongliang, C.: On Global Performance Indices of Robotic Mechanisms, Robot, 27, 420-424, 2005.

Zhou, Y., Niu, J., Liu, Z., and Zhang, F.: A novel numerical approach for workspace determination of parallel mechanisms, J. Mech. Sci. Technol., 31, 3005-3015, 2017.

Zlatanov, D., Fenton, R. G., and Benhabib, B.: A Unifying Framework for Classification and Interpretation of Mechanism Singularities, J. Mech. Design, 117, 566, https://doi.org/10.1115/1.2826720, 1995.

Zlatanov, D., Bonev, I. A., and Gosselin, C. M.: Constraint singularities of parallel mechanisms, 496-502, IEEE International Conference of Robotics and Automation (ICRA), Washington, DC, USA, 2002. 